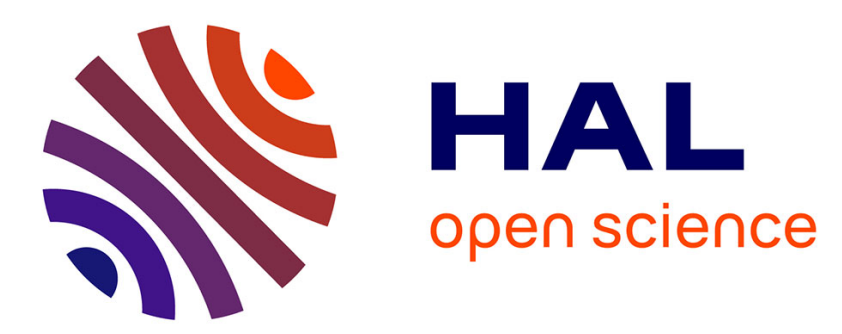

\title{
Mesoscale probabilistic models for the elasticity tensor of fiber reinforced composites: Experimental identification and numerical aspects
}

\author{
Johann Guilleminot, Christian Soize, D. Kondo
}

\section{- To cite this version:}

Johann Guilleminot, Christian Soize, D. Kondo. Mesoscale probabilistic models for the elasticity tensor of fiber reinforced composites: Experimental identification and numerical aspects. Mechanics of Materials, 2009, 41 (12), pp.1309-1322. 10.1016/j.mechmat.2009.08.004 hal-00684330

\author{
HAL Id: hal-00684330 \\ https://hal.science/hal-00684330
}

Submitted on 1 Apr 2012

HAL is a multi-disciplinary open access archive for the deposit and dissemination of scientific research documents, whether they are published or not. The documents may come from teaching and research institutions in France or abroad, or from public or private research centers.
L'archive ouverte pluridisciplinaire HAL, est destinée au dépôt et à la diffusion de documents scientifiques de niveau recherche, publiés ou non, émanant des établissements d'enseignement et de recherche français ou étrangers, des laboratoires publics ou privés. 


\title{
Mesoscale probabilistic models for the elasticity tensor of fiber reinforced composites: experimental identification and numerical aspects.
}

\author{
Johann Guilleminot ${ }^{\mathrm{a}, *}$, Christian Soize $^{\mathrm{a}}$, Djimedo Kondo ${ }^{\mathrm{b}}$ \\ ${ }^{a}$ Université Paris-Est, Laboratoire Modélisation et Simulation Multi Echelle, MSME \\ FRE3160 CNRS, 5 Bd Descartes, 77454 Marne la Vallée, France \\ ${ }^{b}$ Laboratoire de Mécanique de Lille-UMR CNRS 8107, Université des Sciences et \\ Technologies de Lille, Cité Scientifique, Bd. Paul Langevin, 59655 Villeneuve d'Ascq \\ Cedex, France
}

\begin{abstract}
This work deals with the computational and experimental identification of two probabilistic models. The first one was recently proposed in the literature and provides a direct stochastic representation of the mesoscopic elasticity tensor random field for anisotropic microstructures. The second one, formulated in this paper, is associated to the volume fraction random field at the mesoscale of reinforced composites. After having defined the probabilistic models, we first address the question of the identification of the experimental trajectories of the random fields. For this purpose, we introduce a new methodology relying on the combination of a non destructive ultrasonic testing with an inverse micromechanical problem. The parameters involved in the probabilistic models are then identified and allows realizations of the random fields to be simulated by using Monte Carlo numerical simulations. A comparison between simulated and experimental results is provided and demonstrates the relevance of the identification strategy for the chaos coefficients involved in the second model. Finally, we illustrate the use of the first probabilistic model by performing a probabilistic parametric analysis of the RVE size of the considered microstructure.
\end{abstract}

Key words: composite materials, probabilistic model, experimental identification, Karhunen-Loeve expansion, Polynomial Chaos expansion.

\footnotetext{
* Corresponding author

Email addresses: johann.guilleminot@univ-paris-est.fr (Johann Guilleminot)
} 


\section{Introduction}

Within a micromechanical framework, the concept of a Representative Volume Element (RVE) has been largely investigated over the past 20 years. While there does not exist a precise definition of the RVE size, a general and commonly used definition of the latter is that it has to be "sufficiently large" with respect the size of the heterogeneities and "sufficiently small" with respect to the size of the structure. When a homogenization procedure is carried out with reference to this concept, one obtains estimates that are (i) considered as effective, in the sense that they represent (in a deterministic way) the behavior of an associated (fictitious) homogeneous solid; (ii) independent of the boundary condition type that is prescribed for the homogenization procedure. Such a homogenization framework is well adapted when the size of the heterogeneities is very small in comparison to the one defining the scale of interest (in industrial applications or experimental testing, for instance). This is usually the case of polycrystalline materials or composites reinforced by micrometric particles.

However, for other kind of materials, such as rock-like materials, concrete or composites reinforced with long (or continuous) fibers, one may not face samples whose size is sufficiently large in order the RVE concept to be used. In this case, the volume under investigation is called Statistical Volume Element (SVE). The associated mechanical properties are said to be apparent and depend on the realization of the random media as well as on the prescribed boundary conditions (see [7], [14] and [16]).

Several approaches have been proposed in the literature in order to take into account the random dimension arising in the mechanics of heterogeneous materials. At the microscale, the most widely used techniques are based on a combination of a statistical analysis, used for a morphological description of the media and generally performed on a set of digitalized microstructures, with numerical Monte-Carlo simulations (MCS) [10] [18] [25]. In some cases however, the identification of probabilistic models at such a scale may be problematic, because of a too noisy representation for instance [1]. Mesoscopic approximations, such as the Moving Window-GMC technique (consisting in the combination of a statistical analysis performed on locally homogenized properties at mesoscale, with MCS; see [1] [5]) may then be considered. While such modeling clearly smoothes some probabilistic information arising at finer scales, it may be more suitable in practice regarding experimental identification (depending also on the case under consideration). Within this framework, another kind of approach consists in constructing probabilistic mathematical models (instead of statistical analysis) which en- 
sure, for instance, some key probabilistic features of the solution associated to the stochastic boundary value problem. Thus, a mesoscopic probabilistic model for random anisotropic elastic microstructures was recently proposed by Soize (see [22] [23]) and used to investigate the RVE size by performing a probabilistic parametric analysis. In particular, it was shown that the characteristic length of the macroscopic scale could be defined with respect to the spatial correlation lengths of the mesoscopic elasticity random field. In this context of stochastic analysis, there are only a very few probabilistic studies based on experimental identification and this lack of physically-based modeling has been pointed out in many state-of-the-art reports in stochastic mechanics [4]. In fact, most of previous analysis were based on assumed probabilistic properties and focused on the uncertainty propagation. This paper is precisely devoted to the computational and experimental identification of two probabilistic models of the elasticity tensor random field at the mesoscale of a fiber reinforced composite. The first model is the probabilistic model for random anisotropic elastic microstructures relying on a minimal parametrization introduced in [22] [23]. In addition to this approach, we further propose a probabilistic model dedicated to the mesoscopic volume fraction in reinforcement, with reference to the one defined in [6] (in the case of the microscopic volume fraction). It relies on two classical representation techniques of random fields (namely, the Karhunen-Loeve and Polynomial Chaos expansions) and allows trajectories of the elasticity tensor to be simulated through a homogenization procedure.

The paper is organized as follows. The two probabilistic models are first defined in section (2). The identification of the parameters involved in the stochastic representations required the development of dedicated experimental methodology and setup. This point is precisely addressed in section (3). Based on this identification, the determination of the experimental trajectories of the random fields (from which some probabilistic features will be characterized), as well as of the parameters involved in the probabilistic models, is carried out. A comparison between the experimental and simulated realizations is also provided and allows one to discuss the relevance of the strategy identification of the chaos coefficients involved in the probabilistic model of the mesoscopic volume fraction. Finally, we illustrate, in section (4), the use of the direct probabilistic model of the elasticity tensor random field by performing a probabilistic parametric analysis of the RVE size with respect to the spatial correlation lengths of the mesoscopic random field. 


\section{Probabilistic models}

\subsection{Probabilistic model of the elasticity tensor random field}

Let us first recall some key features of the mesoscale probabilistic model for random anisotropic elastic microstructures introduced in [22] [23]. Let $\mathbf{x} \rightarrow \mathbb{C}(\mathbf{x})$ be the fourth-order tensor-valued random field corresponding to this model. In the sequel, we will consider the matrix representation $\mathbf{C}(\mathbf{x})$ of the elasticity tensor $\mathbb{C}(\mathbf{x})^{1}$. For a given $\mathbf{x}$ in $\Omega, \mathbf{C}(\mathbf{x})$ is a random variable with values in $\mathbb{M}_{n}^{+}(\mathbb{R})$ (the set of all the $n \times n$ real symmetric positive-definite matrices). Let $\mathbf{x} \rightarrow \underline{\mathbf{C}}(\mathbf{x})$ be the mean function of $\mathbf{C}(\mathbf{x})$ which is assumed to be known. For a given $\mathbf{x}$ in $\Omega$, let us introduce the normalized random field $\mathbf{x} \rightarrow \mathbf{G}(\mathbf{x})$, defined on a probability space $(\Theta, \mathcal{F}, P)$, indexed by $\mathbb{R}^{d}$ and with values in $\mathbb{M}_{n}^{+}(\mathbb{R})$ (in the most general case, $d=3$ and $n=6$ ), such that:

$$
\left\{\begin{array}{l}
\mathbf{C}(\mathbf{x})=\underline{\mathbf{L}}(\mathbf{x})^{\mathrm{T}} \mathbf{G}(\mathbf{x}) \underline{\mathbf{L}}(\mathbf{x}) \\
E\{\mathbf{G}(\mathbf{x})\}=\mathbf{I}
\end{array}\right.
$$

where $\mathbf{I}$ is the $n \times n$ identity matrix and $\underline{\mathbf{L}}(\mathbf{x})$ is the upper triangular matrix arising from the Cholesky factorization of the mean matrix $(\underline{\mathbf{C}}(\mathbf{x})=$ $\left.\underline{\mathbf{L}}(\mathbf{x})^{\mathrm{T}} \underline{\mathbf{L}}(\mathbf{x})\right)$.

\subsubsection{Construction of the probability density function of random matrix $\boldsymbol{G}(\boldsymbol{x})$}

For a given $\mathbf{x}$, the probability distribution $P_{\mathbf{G}(\mathbf{x})}$ of random matrix $\mathbf{G}(\mathbf{x})$ is defined by a probability density function $\mathbf{G} \rightarrow p_{\mathbf{G}(\mathbf{x})}(\mathbf{G})$ such that $P_{\mathbf{G}(\mathbf{x})}=p_{\mathbf{G}(\mathbf{x})}(\mathbf{G}) \widetilde{d} \mathbf{G}$ with respect to $\widetilde{d} \mathbf{G}=2^{n(n-1) / 4} \prod_{1 \leq i \leq j \leq n} d \mathbf{G}_{i j}$ (where $d \mathbf{G}_{i j}$ is the Lebesgue measure on $\mathbb{R}$ ). The probability density function can be constructed by using the Maximum Entropy Principle (see [8] [9]). The approach relies on the notion of Entropy introduced by Shannon in the context of Information Theory ([19]) and allows the probabilistic model to be constructed with respect to the available information. It can then be shown that:

$$
p_{\mathbf{G}(\mathbf{x})}(\mathbf{G})=\mathbf{1}_{\mathbb{M}_{n}^{+}(\mathbb{R})}(\mathbf{G}) c_{G}(\operatorname{det}(\mathbf{G}))^{(n+1)\left(1-\delta^{2}\right) /\left(2 \delta^{2}\right)} \exp \left\{-\frac{n+1}{2 \delta^{2}} \operatorname{tr}(\mathbf{G})\right\}
$$

where $\mathbf{G} \rightarrow \mathbf{1}_{\mathbb{M}_{n}^{+}(\mathbb{R})}(\mathbf{G})=1$ if $\mathbf{G} \in \mathbb{M}_{n}^{+}(\mathbb{R})$ (=0 otherwise), $c_{G}$ is a positive constant (whose expression is provided in [23]) and $\delta$ is a parameter that will

\footnotetext{
${ }^{1}$ The mapping is defined as follows: $\mathbf{C}(\mathbf{x})_{I J}=\mathbb{C}_{i j k l}(\mathbf{x}),(I, J) \in(1 . .6)^{2}$ with $I=(i, j)$, $J=(k, l)$ and where $1=(1,1), 2=(2,2), 3=(3,3), 4=(1,2), 5=(1,3), 6=(2,3)$.
} 
be defined below; det and tr are the determinant and the trace of matrices, respectively.

From an algebraic standpoint, the normalized random field $\mathbf{x} \rightarrow \mathbf{G}(\mathbf{x})$ is defined as the non-linear mapping of $n(n+1) / 2$ second-order centered homogeneous Gaussian random fields $\mathbf{x} \rightarrow \mathbf{U}_{j j^{\prime}}(\mathbf{x})$ (with $1 \leq j \leq j^{\prime} \leq n$ ), defined on $(\Theta, \mathcal{F}, P)$, indexed by $\mathbb{R}^{d}$ and with values in $\mathbb{R}$. The explicit expression of this non-linear mapping can be found in [22] [23]. The stochastic germs are such that for all $\mathbf{x}$ in $\mathbb{R}^{d}, E\left\{\mathbf{U}_{j j^{\prime}}(\mathbf{x})\right\}=0$ and $E\left\{\mathbf{U}_{j j^{\prime}}(\mathbf{x})^{2}\right\}=1$ and are then defined by the $n(n+1) / 2$ autocorrelation functions $R_{\mathbf{U}_{j j^{\prime}}}(\tau)=$ $E\left\{\mathbf{U}_{j j^{\prime}}(\mathbf{x}+\tau) \mathbf{U}_{j j^{\prime}}(\mathbf{x})\right\}$ (with $\tau \in \mathbb{R}^{d}$ and $R_{\mathbf{U}_{j j^{\prime}}}(0)=1$ ). A minimal parametrization can be obtained by choosing $R_{\mathbf{U}_{j j^{\prime}}}(\tau)=\prod_{k=1}^{d} r_{j j^{\prime}}^{k}\left(\tau_{k}\right)$ with $r_{j j^{\prime}}^{k}\left(\tau_{k}\right)=4\left(l_{j j^{\prime}}^{k}\right)^{2} / \pi^{2} \tau_{k}^{2} \cdot \sin ^{2}\left(\pi \tau_{k} / 2 l_{j j^{\prime}}^{k}\right)$ and where the spatial correlation lengths $\left\{l_{j j^{\prime}}^{k}\right\}$ are real parameters of the model. The definition of the normalized random field also depends on a real parameter $\delta$ (with $0<\delta<\sqrt{(n+1) /(n+5)})$, independent of $\mathbf{x}$ and $n$, such that:

$$
\delta=\sqrt{\frac{1}{n} E\left\{\|\mathbf{G}(\mathbf{x})-\mathbf{I}\|_{\mathrm{F}}^{2}\right\}}
$$

where $\|\cdot\|_{F}$ is the Frobenius norm. This parameter allows the statistical fluctuations of $\mathbf{x} \rightarrow \mathbf{G}(\mathbf{x})$ to be controlled. It can also be shown that $E\left\{\|\mathbf{G}(\mathbf{x})\|_{\mathrm{F}}^{2}\right\}=n\left(\delta^{2}+1\right)$. Finally, let $\delta_{\mathbf{C}}(\mathbf{x})$ be the parameter defined by:

$$
\delta_{\mathbf{C}}(\mathbf{x})=\frac{E\left\{\|\mathbf{C}(\mathbf{x})-\underline{\mathbf{C}}(\mathbf{x})\|_{\mathrm{F}}^{2}\right\}}{\|\underline{\mathbf{C}}(\mathbf{x})\|_{\mathrm{F}}^{2}}
$$

It can be shown that $\delta_{\mathbf{C}}(\mathbf{x})=\delta / \sqrt{n+1} \cdot\left\{1+(\operatorname{tr}(\underline{\mathbf{C}}(\mathbf{x})))^{2} / \operatorname{tr}(\underline{\mathbf{C}}(\mathbf{x}))^{2}\right\}^{1 / 2}$. Further details about the definition of random field $\mathbf{x} \rightarrow \mathbf{G}(\mathbf{x})$ can be found in the references given above.

In the homogeneous case, the dispersion parameter (3) does not depend on $\mathbf{x}$ anymore and the model is then completely defined by (i) the mean matrix $\underline{\mathbf{C}}$ (ii) the dispersion parameter (either $\delta$ or $\delta_{\mathbf{C}}$ ) (iii) the spatial correlation lengths of the stochastic germs. Let $\tau=\left(\tau_{x}, \tau_{y}, \tau_{z}\right) \rightarrow r^{\mathbf{C}}(\tau)$ be the function defined from $\mathbb{R}^{3}$ into $\mathbb{R}$ by:

$$
r_{\mathbf{C}}(\tau)=\frac{\operatorname{tr} E\{(\mathbf{C}(\mathbf{x}+\tau)-\underline{\mathbf{C}})(\mathbf{C}(\mathbf{x})-\underline{\mathbf{C}})\}}{E\left\{\|\mathbf{C}(\mathbf{x})-\underline{\mathbf{C}}\|_{\mathrm{F}}^{2}\right.}
$$


The spatial correlation length of random field $\mathbf{x} \rightarrow \mathbf{C}(\mathbf{x})$ associated with direction $u(u=x, y$ or $z)$ is then given by $l_{\mathbf{C}}^{u}=\int_{0}^{+\infty}\left|r_{\mathbf{C}}\left(\tau^{u}\right)\right| d \tau_{u}$ (with $\tau^{x}=\left(\tau_{x}, 0,0\right), \tau^{y}=\left(0, \tau_{y}, 0\right)$ and $\left.\tau^{z}=\left(0,0, \tau_{z}\right)\right)$.

\subsubsection{Fundamental property of the normalized random field}

Let $\Omega$ be any bounded open domain of $\mathbb{R}^{d}$ and let $\bar{\Omega}$ be its closure. It can be shown that:

$$
E\left\{\left(\sup _{\mathbf{x} \in \bar{\Omega}}\left\|\mathbf{G}(\mathbf{x})^{-1}\right\|\right)^{2}\right\}<+\infty
$$

in which $\|\cdot\|$ denotes the operator norm. Eq. (5) allows one to define a non-uniform ellipticity condition for the random bilinear form involved in the weak formulation of the associated stochastic boundary value problem.

\subsubsection{Numerical simulations of the stochastic germs [22]}

Let $\mathbf{V}_{j j^{\prime}}$ be the vectorial representation of stochastic germ $\mathbf{U}_{j j^{\prime}}$, i.e. $\mathbf{V}_{j j^{\prime}}=\left(\mathbf{U}_{j j^{\prime}}\left(\mathbf{x}_{1}\right), \ldots, \mathbf{U}_{j j^{\prime}}\left(\mathbf{x}_{N}\right)\right)$. The numerical technique for simulating homogeneous Gaussian vector-valued random fields has been largely addressed in the litterature (see the pioneering work from Shinozuka [20], as well as [15] for instance) and is recalled in the sequel. For the sake of simplicity, let us consider simulations in a given principal direction (i.e. $d=1$ ) and the case of the correlation function introduced in section (2.1.1). The $p^{s i m}$-order approximation of vector $\mathbf{V}_{j j^{\prime}}$ then reads [15]:

$$
\mathbf{V}_{j j^{\prime}}^{p^{s i m}}=\sum_{i=1}^{p^{s i m}} \sqrt{2 S_{i}} \sqrt{-\log \left(\psi_{i}\right)} \cos \left(\Phi_{i}+\frac{\pi}{l_{j j^{\prime}}^{k}} \chi_{i} \mathbf{x}^{k}\right)
$$

where $l_{j j^{\prime}}^{k}$ is the spatial correlation length of the germ and $\mathbf{x}^{k}$ is the vector gathering the coordinates of the points, both associated to the considered direction $k$ (in case the germ is simulated in the $\mathrm{x}$-direction, one has $l_{j j^{\prime}}^{k}=l_{j j^{\prime}}^{x}$ and $\mathbf{x}^{k}=\left(x_{1}, \ldots, x_{N}\right)$ for instance). For $i \in\left\{1, \ldots, p^{s i m}\right\}$, one further defines $S_{i}=\amalg\left(\chi_{i}\right)$ and $\chi_{i}=-1+2\left(i-\frac{1}{2}\right) / p^{\text {sim }}$, where $\chi \rightarrow \amalg(\chi)$ is the continuous function from $\mathbb{R}$ into $\mathbb{R}^{+}$, with compact support $[0,1]$, such that $\amalg(\chi)=1-\chi$ if $\chi \in[0,1]$ and $\amalg(-\chi)=\amalg(\chi)$. The $N$-dimensional vector $\Phi_{i}$ is such that $\Phi_{i}=\left(\phi_{i}, \ldots, \phi_{i}\right)$. The two sets of independent random variables $\left\{\psi_{i}\right\}_{i=1}^{p^{s i m}}$ and $\left\{\phi_{i}\right\}_{i=1}^{p^{s i m}}$ are such that $\psi_{i}$ and $\phi_{i}$ are uniformly distributed re-

spectively on $[0,1]$ and $[0,2 \pi]$. It can then be shown that $\mathbf{V}_{j j^{\prime}}^{p^{s i m}}$ is a Gaussian random vector whose covariance matrix converges to the covariance matrix 
of $\mathbf{V}_{j j^{\prime}}$ when $p^{\text {sim }}$ goes to infinity.

Remark:

For moderate values of $N$, simulations of $\mathbf{V}_{j j^{\prime}}$ can be readily obtained by writting the random vector as $\mathbf{V}_{j j^{\prime}}=\left[L_{\mathbf{V}}\right]^{\mathrm{T}} \tilde{\mathbf{V}}$, where $\left[L_{\mathbf{V}}\right]$ is the upper triangular matrix arising from the Cholesky factorization of the target covariance matrix and $\widetilde{\mathbf{V}}$ is a random vector whose components are independent normalized Gaussian random variables. It should be mentionned that when the random field has to be simulated on a fine grid, the covariance matrix (which is a full matrix) becomes very large and in addition, it may become ill-conditioned for the cholesky decomposition.

In some particular cases, the randomness exhibited by the mesoscopic elasticity tensor random field may be mainly induced by a given microstructural parameter, such as volume fraction, fiber spatial distribution or orientation. In this context, it is of interest to construct a probabilistic model of this mesoscopic microstructural parameter. In the next section, we present such a model, based on the one introduced in [6], the microstructural parameter being the fiber volume fraction.

\subsection{Parametric probabilistic model of the mesoscopic volume fraction ran- dom field}

Let $\mathbf{x} \rightarrow f(\mathbf{x})$ be a random field defined on a probability space $(\Theta, \mathcal{F}, \mathcal{P})$, indexed by a bounded set $\Omega$ in $\mathbb{R}^{2}$ with values in $[0,1]$. It is assumed that $f$ is a second-order random field and is such that $\int_{\Omega} \int_{\Omega}\left|\mathrm{C}_{f}\left(\mathbf{x}, \mathbf{x}^{\prime}\right)\right|^{2} d \mathbf{x} d \mathbf{x}^{\prime}<+\infty$, where $\left(\mathbf{x}, \mathbf{x}^{\prime}\right) \rightarrow \mathrm{C}_{f}\left(\mathbf{x}, \mathbf{x}^{\prime}\right)$ is the covariance function of the random field, defined from $\Omega \times \Omega$ into $\mathbb{R}$. Let us consider the truncated Karhunen-Loeve expansion of random field $f$ (see [13]):

$$
f(\mathbf{x})=\underline{f}(\mathbf{x})+\sum_{k=1}^{M} \sqrt{\lambda_{k}} \eta_{k} \psi_{k}(\mathbf{x})
$$

where $\mathbf{x} \rightarrow \underline{f}(\mathbf{x})$ denotes the mean function; $\left\{\lambda_{k}\right\}_{k=1}^{M}$ and $\left\{\psi_{k}\right\}_{k=1}^{M}$ are the sets of eigenvalues and eigenfunctions of the linear integral operator with covariance kernel, satisfying the integral equation:

$$
\int_{\Omega} \mathrm{C}_{f}\left(\mathbf{x}, \mathbf{x}^{\prime}\right) \psi_{k}\left(\mathbf{x}^{\prime}\right) d \mathbf{x}^{\prime}=\lambda_{k} \psi_{k}(\mathbf{x})
$$

The set $\left\{\eta_{k}\right\}_{k=1}^{M}$ is a set of random variables such that $E\left\{\eta_{i}\right\}=0$ and $E\left\{\eta_{i} \eta_{j}\right\}=\delta_{i j}$, where $E$ is the mathematical expectation and $\delta_{i j}$ is the Kro- 
necker symbol. The orthogonality of the basis $\left\{\psi_{k}\right\}_{k=1}^{M}$ allows independent realizations of the random variables to be computed from Eq. (7):

$$
\eta_{i}\left(\theta_{j}\right)=\frac{1}{\sqrt{\lambda_{i}}}<f\left(\theta_{j}\right)-\underline{f}, \psi_{i}>_{\mathcal{L}^{2}(\Omega, \mathbb{R})}
$$

where $<\cdot, \cdot>_{\mathcal{L}^{2}(\Omega, \mathbb{R})}$ is the inner product on Hilbert space $\mathcal{L}^{2}(\Omega, \mathbb{R})$. Next, gathering the random variables $\eta_{1}, \ldots, \eta_{M}$ in a single random vector $\eta$, let us consider the $q$-order polynomial Gaussian chaos expansion of $\eta$ [3] [24] [26]:

$$
\eta=\sum_{\gamma \in \mathbb{N}^{m},|\gamma|=1}^{q} z^{\gamma} \tilde{H}_{\gamma}(\xi)
$$

where $\tilde{H}_{\gamma}(\xi)=H_{\gamma}(\xi) / \sqrt{\gamma !}\left(\right.$ with $|\gamma|=\sum_{k=1}^{m} \gamma_{k}$ and $\left.\gamma !=\prod_{k=1}^{m} \gamma_{k} !\right),\left\{H_{\gamma}\right\}_{\gamma}$ are multivariate Hermite polynomials, orthogonal with respect to the canonical Gaussian probability measure; $\left\{z^{\gamma}\right\}_{\gamma}$ is a set of deterministic vectors in $\mathbb{R}^{M}$. The germ $\xi$ is a $m$-dimensional zero-mean Gaussian vector such that $E\left\{\xi_{i} \xi_{j}\right\}=\delta_{i j}$. The representation of random field $f$ then reads:

$$
f(\mathbf{x})=\underline{f}(\mathbf{x})+\sum_{k=1}^{M} \sqrt{\lambda_{k}}\left\{\sum_{\gamma \in \mathbb{N}^{m},|\gamma|=1}^{q} z_{k}^{\gamma} \tilde{H}_{\gamma}(\xi)\right\} \psi_{k}(\mathbf{x})
$$

where $z_{k}^{\gamma}$ is the $k$-th component of vector $z^{\gamma}$. From Eq. (11), it must be emphasized that the identification of the random field consists in the identification of the deterministic coefficients involved in the chaos representation. It should be noted that such an identification can be a major practical difficulty. For this purpose, let $\mathbf{Z}$ be the $M \times Q$ matrix whose columns are the vectors $z^{\gamma}$ (where $Q$ is the number of terms involved in the chaos expansion). Let $\left\{\Xi^{1}, \Xi^{2}, \ldots, \Xi^{p^{e x p}}\right\}$ be $p^{e x p}$ experimental realizations of the random vector $\eta$ (with $\Xi_{j}^{i}=\eta_{j}\left(\theta_{i}\right)$ ). It can be shown that the orthogonality of the random variables involved in the Karhunen-Loeve expansion yields the following algebraic constraint:

$$
\mathbf{Z} \mathbf{Z}^{T}=\mathbf{I}_{M}
$$

where $\mathbf{I}_{M}$ is the $M \times M$ identity matrix. The identification of $\mathbf{Z}$ can be achieved by using the formulation proposed in [2] which is based on the use of the Maximum Likelihood Method [17] and which then consists in solving the following optimization problem :

$$
\max \mathrm{L}\left(\Xi^{1}, \ldots, \Xi^{p^{e x p}} ; \mathbf{Z}\right)
$$


with

$$
\mathrm{L}\left(\Xi^{1}, \ldots, \Xi^{p^{e x p}} ; \mathbf{Z}\right)=\sum_{i=1}^{p^{e x p}} \sum_{j=1}^{M} \log _{10}\left\{p_{\eta_{j}}\left(\Xi_{j}^{i}, \mathbf{Z}\right)\right\}
$$

under the constraint (12). The problem (13) can be solved by a random search strategy, i.e. by randomly sampling the manifold defined by Eq. (12) and by selecting the realization which maximizes the pseudo log-likelihood function (14). The values of the marginal distributions can be computed by using, for instance, a classical kernel estimate (see [21] for a survey):

$$
\widehat{p}_{\eta_{j}}\left(\Xi_{j}^{i}, \mathbf{Z}\right)=\frac{1}{N h} \sum_{k=1}^{N} \mathrm{~K}\left(\frac{\Xi_{j}^{i}-\Xi_{j}^{\operatorname{sim}_{j}^{k}}}{h}\right)
$$

where $N$ and $h$ are the number of simulation of the Gaussian germ $\xi$ and

the bandwith respectively, $\Xi^{\operatorname{sim}^{k}}$ is the $j$-th component of the $k$-th simulated realization of random vector $\eta$ (computed by using Eq. (10) for a given $\mathbf{Z}$ ) and $x \rightarrow \mathrm{K}(x)$ is the kernel function, defined on $\mathbb{R}$ and such that $\forall x \in$ $\mathbb{R}, \mathrm{K}(x) \geqslant 0$ and $\int_{\mathbb{R}} \mathrm{K}(x) d x=1$. In this study, a Gaussian kernel function is used, $\mathrm{K}(x)=1 / \sqrt{2 \pi} \exp \left(-1 / 2 \cdot x^{2}\right)$.

\section{Computational and experimental identification}

In this study, we consider the case of thin composite plates (with dimensions $158 \times 98 \times 3 \mathrm{~mm}$ ), made up of a thermoplastic matrix (polypropylene) reinforced by E-glass long fibers (with a target volume fraction after processing: $f^{\text {tgt }}=10 \%$ ). Details about the experimental setup can be found in Guilleminot et al. [6].

\subsection{Definition of a mechanical inverse problem and identification of the experimental trajectories}

We now formulate the methodology that allows the experimental trajectories of random fields $\mathbf{x} \rightarrow f(\mathbf{x})$ and $\mathbf{x} \rightarrow \mathbf{C}(\mathbf{x})$ to be identified. Let us recall first that the velocity of longitudinal waves propagating inside a isotropic elastic medium can be written as:

$$
V_{l}=\sqrt{\frac{E(1-\nu)}{\rho(1+\nu)(1-2 \nu)}}
$$

where $\rho, E$ and $\nu$ are the density, the Young's modulus and Poisson ratio of the material, respectively. Let $(E, \nu, \rho) \rightarrow V_{l}=\mathrm{A}(E, \nu, \rho)$ be the function 
defined by Eq. (16). Furthermore, since some microstructural features of the medium are known a priori, a multi-scale analysis allows one to express the mechanical properties (the density being defined by a rule of mixture) as a function of the volume fraction in reinforcement inside the volume that is considered. Let $f \rightarrow(E, \nu)=\mathrm{M}(f)$ be the function defining such a mapping. Finally, let $f \rightarrow V_{l}=\Lambda(f)$ be the composed function $\Lambda=\mathrm{A} \circ \mathrm{M}$, where both equivalence between dynamic and static moduli and local isotropy are assumed. Note that for the sake of brevity, the mapping $M$ then implicitely integrates (by abuse of notation) the definition of the density. The function $\Lambda$ allows the longitudinal velocity to be predicted from the volume fraction in reinforcement, as summarized on Fig. (1). The experimental realizations

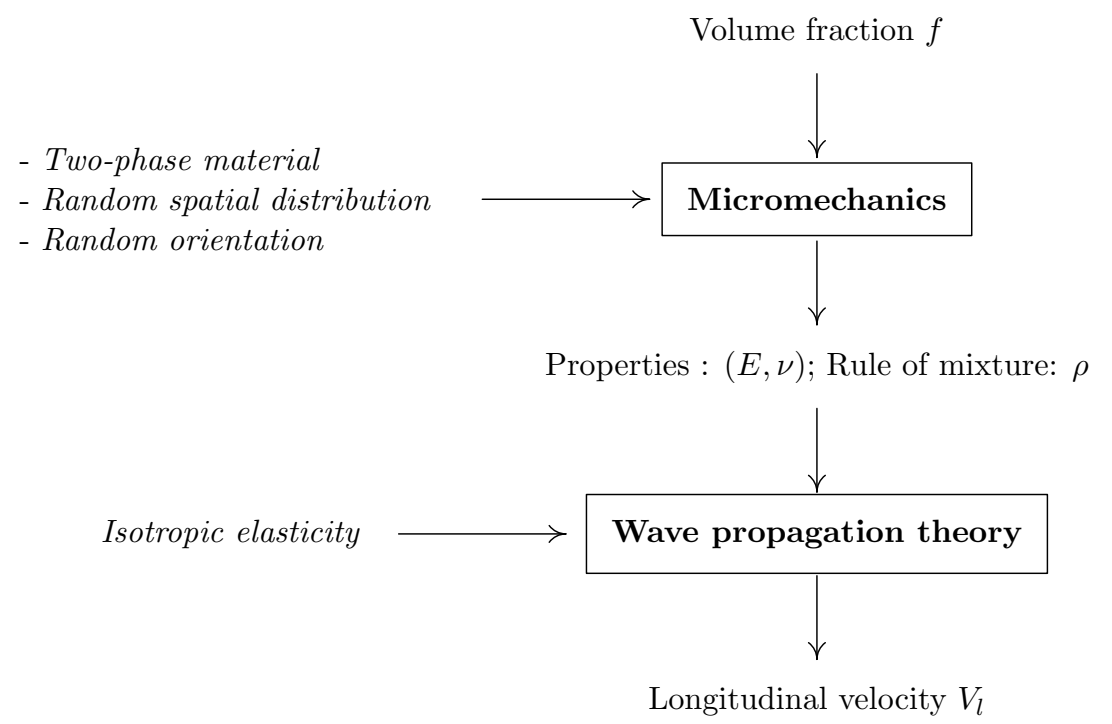

Figure 1: Scheme of the direct mechanical problem.

of random fields $\mathbf{x} \rightarrow f(\mathbf{x})$ and $\mathbf{x} \rightarrow \mathbf{C}(\mathbf{x})$ can then be determined by constructing the inverse mapping $V_{l} \rightarrow f=\Lambda^{-1}\left(V_{l}\right)$, defined from $S_{\Lambda} \subset \mathbb{R}^{+}$ into $[0,1]$. This construction can be numerically achieved provided that function $\mathrm{M}$ is known. From a micromechanical standpoint, the mesoscopic approximation implies this function to be random (see the references [7], [14] and [16]), so that the mapping is not one-to-one and $\Lambda^{-1}$ can not be defined. However, because the statistical fluctuations are mainly generated by fiber clustering (roughly speaking, the volume fraction in the mesovol- 
umes is either small or large), we assume that the randomness is only due to volume fraction fluctuations, rather than to fluctuations of any other microstructural parameter (orientation of the fibers, etc.). In other words, the local material symmetry is preserved over all locations, and we may now consider a given realization of the random function, $\mathrm{M}_{k}=\mathrm{M}\left(\theta_{k}\right)$. In this very particular context, it still remains to choose the mechanical solver to be used for estimating $\mathrm{M}_{k}$. Using a computational procedure would require in the present case the $3 \mathrm{D}$ analysis of volumes containing tens of thousands of fibers, leading to a prohibitive computational effort when no simplifying assumptions are made. Thus, it is proposed to use a mean-field homogenization technique (more precisely, the Mori-Tanaka scheme) in order to identify the function M. While this choice is arbitrary (note however that the micromechanical scheme was choosen according to usual micromechanical considerations, such as the "matrix-inclusion" morphology of the composite), it benefits from its simple formulation. A discussion about this procedure is provided in [6] (the plot of the mapping $V_{l} \rightarrow f=\Lambda^{-1}\left(V_{l}\right)$ is depicted in Appendix A). Finally, let $\mathcal{V}^{\text {exp }}$ be the set of experimental results obtained by the ultrasonic testing:

$$
\mathcal{V}^{e x p}=\left\{V_{l}^{e x p}\left(\mathbf{x}_{i}, \theta_{j}\right), i \in\left[1, \ldots, N^{e x p}\right], j \in\left[1, \ldots, p^{e x p}\right]\right\}
$$

Let $\mathcal{S}^{\exp }$ be the set corresponding to the experimental realizations of the mesoscopic volume fraction random field, defined by:

$$
\mathcal{S}^{\exp }=\left\{f^{\exp }\left(\mathbf{x}_{i}, \theta_{j}\right), i \in\left[1, \ldots, N^{\exp }\right], j \in\left[1, \ldots, p^{\exp }\right]\right\}
$$

where for all $(i, j)$ in $\left[1, \ldots, N^{\exp }\right] \times\left[1, \ldots, p^{\exp }\right]\left(\right.$ with $\left.p^{\exp }=110\right)$ :

$$
f^{e x p}\left(\mathbf{x}_{i}, \theta_{j}\right)=\Lambda^{-1}\left(V_{l}^{e x p}\left(\mathbf{x}_{i}, \theta_{j}\right)\right)
$$

In the same way, let $\mathcal{C}^{\exp }$ be the set corresponding the experimental trajectories of the mesoscopic elasticity tensor random field, defined with respect to $\mathcal{S}^{\exp }$ by the following mapping:

$$
\mathcal{C}^{e x p}=\left\{\mathbf{C}^{e x p}\left(\mathbf{x}_{i}, \theta_{j}\right), i \in\left[1, \ldots, N^{e x p}\right], j \in\left[1, \ldots, p^{e x p}\right]\right\}
$$

with

$$
\mathbf{C}^{e x p}\left(\mathbf{x}_{i}, \theta_{j}\right)=\mathrm{M}\left(f^{e x p}\left(\mathbf{x}_{i}, \theta_{j}\right)\right)
$$

The set $\mathcal{S}^{\exp }$ (resp. $\mathcal{C}^{\exp }$ ) will be used in section (3.3) (resp. section (3.2)) in order to identity the probabilistic model of the volume fraction (resp. elasticity tensor) random field. The velocity random field was sampled on 
a grid composed of respectively 12 and 7 points in the directions $x$ and $y$, so that $N^{e x p}=84$. It is worth noticing that in the case of the volume fraction, the proposed methodology allows the sampling of $84 \times 110=9240$ mesovolumes. Clearly, such a result would not have been possible by using a classical technique (e.g. a resin burn-off).

The first experimental realization of random fields $\mathbf{x} \rightarrow f^{\exp }(\mathbf{x}), \mathbf{x} \rightarrow$ $\mathbf{C}_{11}^{\exp }(\mathbf{x})$ and $\mathbf{x} \rightarrow \mathbf{C}_{12}^{\exp }(\mathbf{x})$ is depicted on Figs (2), (3) and (4) respectively.

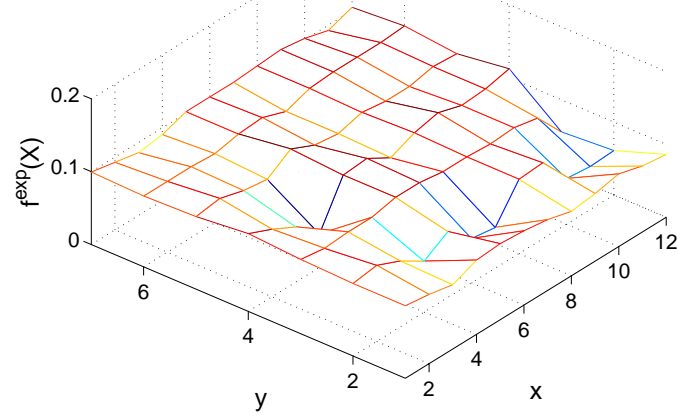

Figure 2: Graph of the first experimental realization $\mathbf{x} \rightarrow f^{\exp }\left(\mathbf{x}, \theta_{1}\right)$.

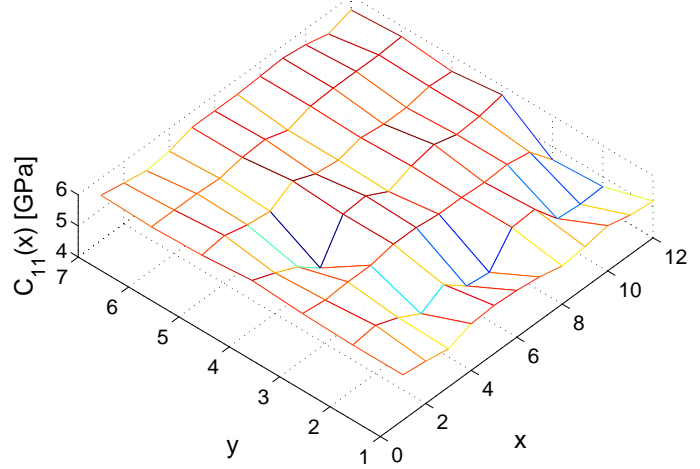

Figure 3: Graph of the first experimental realization $\mathbf{x} \rightarrow \mathbf{C}_{11}^{\exp }\left(\mathbf{x}, \theta_{1}\right)$. 


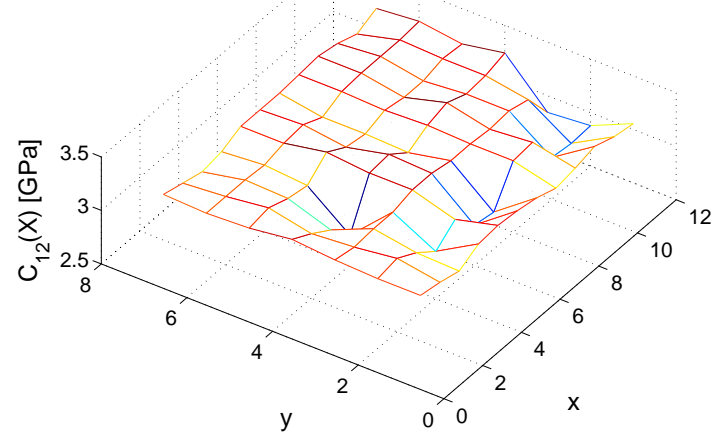

Figure 4: Graph of the first experimental realization $\mathbf{x} \rightarrow \mathbf{C}_{12}^{\exp }\left(\mathbf{x}, \theta_{1}\right)$.

These figures clearly illustrate the spatial fluctuations of both the mesoscopic volume fraction and elastic properties.

\subsection{Identification of the probabilistic model of the elasticity tensor random field}

In this section, we are interested in the identification of the parameters involved in the probabilistic model of random field $\mathbf{x} \rightarrow \mathbf{C}(\mathbf{x})$ (see section $(2.1)$ ), namely the mean matrix $\underline{\mathbf{C}}$, the fluctuation parameter $\delta_{\mathbf{C}}$ (or equivalently, $\delta$ ) as well as the spatial correlation lengths of the stochastic germs $\left\{l_{j j^{\prime}}^{k}\right\}$.

\subsubsection{Identification of the mean matrix}

The mean matrix can be computed from the set $\mathcal{C}^{\exp }$ (see section (3.1)) by using the classical statistical estimate, that is to say:

$$
\underline{\widehat{\mathbf{C}}} \approx \frac{1}{N^{e x p} p^{e x p}} \sum_{i=1}^{N^{e x p}} \sum_{j=1}^{p^{e x p}} \mathbf{C}^{\exp }\left(\mathbf{x}_{i}, \theta_{j}\right)
$$

where $p^{e x p}=110$. This yields:

$$
\widehat{\mathbf{C}}=\left[\begin{array}{cccccc}
5.33 & 3.11 & 3.11 & 0 & 0 & 0 \\
3.11 & 5.33 & 3.11 & 0 & 0 & 0 \\
3.11 & 3.11 & 5.33 & 0 & 0 & 0 \\
0 & 0 & 0 & 1.11 & 0 & 0 \\
0 & 0 & 0 & 0 & 1.11 & 0 \\
0 & 0 & 0 & 0 & 0 & 1.11
\end{array}\right] \text { (in GPa) }
$$


3.2.2. Identification of the spatial correlation lengths of the stochastic germs

The spatial correlation lengths $l_{\mathbf{C}}^{x}$ and $l_{\mathbf{C}}^{y}$ of the mesoscopic random field can be estimated by approximating the correlation function by an exponential-type correlation function, that is to say by solving the following optimization problem:

$\operatorname{Arg} \min _{l_{k} \in \mathbb{R}^{+}} \sum_{i=1}^{N} \sum_{j=1}^{N}\left|r_{\mathbf{C}}^{\operatorname{sim}}\left(0, \ldots, x_{k}^{i}-x_{k}^{j}, \ldots, 0\right)-\exp \left(-\left(x_{k}^{i}-x_{k}^{j}\right) / l_{\mathbf{C}}^{k}\right)\right|$

for the correlation length $l_{\mathbf{C}}^{k}(k=x$ or $y)$. Here, the summation is performed for $x_{k}^{i}-x_{k}^{j} \geq 0$ (where $x_{k}^{i}$ is the $k$-th coordinate of point $x^{i}, 1 \leq k \leq 3$ ) and $\tau \rightarrow r_{\mathbf{C}}^{\operatorname{sim}}(\tau)$ is the correlation function defined by Eq. (4). This optimization problem is solved by means of a stochastic pattern search algorithm (see for instance [11] [12]). For each correlation length, the robustness of the solution with respect to the initial guess of the algorithm is checked (in both cases, four initializations have been tested: $10,20,30,80 \mathrm{~mm}$ ). The convergence of the optimization algorithm is depicted on Fig. (5), in the case of $l_{\mathbf{C}}^{x}$ (here, it should be mentionned that the algorithm converges quickly because the initial guess is very close to the solution). Finally, the mesoscopic spatial
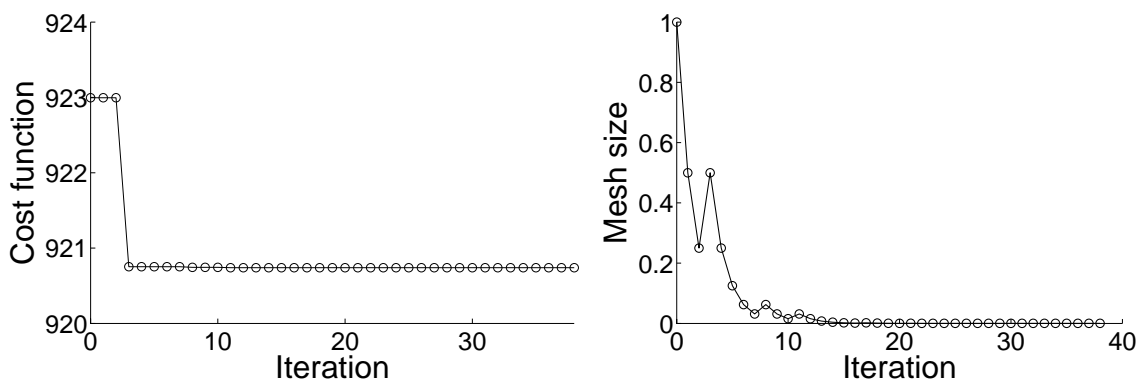

Figure 5: Convergence of the pattern search algorithm, case of $l_{\mathbf{C}}^{x}$ with an initial guess $l^{0}=10 \mathrm{~mm}$ : cost function (left) and adaptive mesh size (right).

correlation lengths are found to be:

$$
\begin{aligned}
& l_{\mathbf{C}}^{x}=11.88 \mathrm{~mm} \\
& l_{\mathbf{C}}^{y}=8.26 \mathrm{~mm}
\end{aligned}
$$

Next, let us assume that the spatial correlation lengths of the stochastic $\operatorname{germs}\left\{l_{j j^{\prime}}^{x}\right\}_{1 \leqslant j \leqslant j^{\prime} \leqslant 6}$ and $\left\{l_{j j^{\prime}}^{y}\right\}_{1 \leqslant j \leqslant j^{\prime} \leqslant 6}$ are such that $\forall\left(j, j^{\prime}\right), l_{j j^{\prime}}^{x}=l_{\mathbf{U}}^{x}$ and 
$l_{j j^{\prime}}^{y}=l_{\mathbf{U}}^{y}$. In order to identify the two correlation lengths $l_{\mathbf{U}}^{x}$ and $l_{\mathbf{U}}^{y}$, it is proposed to numerically construct (for a given direction) the mapping $l_{\mathbf{U}} \rightarrow l_{\mathbf{C}}$ and then to inverse this relation by considering the estimates (24) and (25). The identification of the mesoscopic correlation lengths implies the random field trajectories to be simulated over a sufficiently fine grid. For this purpose, the technique introduced in section (2.1.3) was used and required the parameter $p^{\text {sim }}$ to be properly choosen ${ }^{2}$. The determination of this order was performed by studying the convergence of the correlation estimate for a target correlation function. The convergence of the correlation estimate with respect to the number of Monte Carlo simulations is plotted on Fig. (6) (for $p^{\text {sim }}=512$ ), while the convergence with respect to $p^{\text {sim }}$ is reported on Fig. (7) (for 20000 simulations).

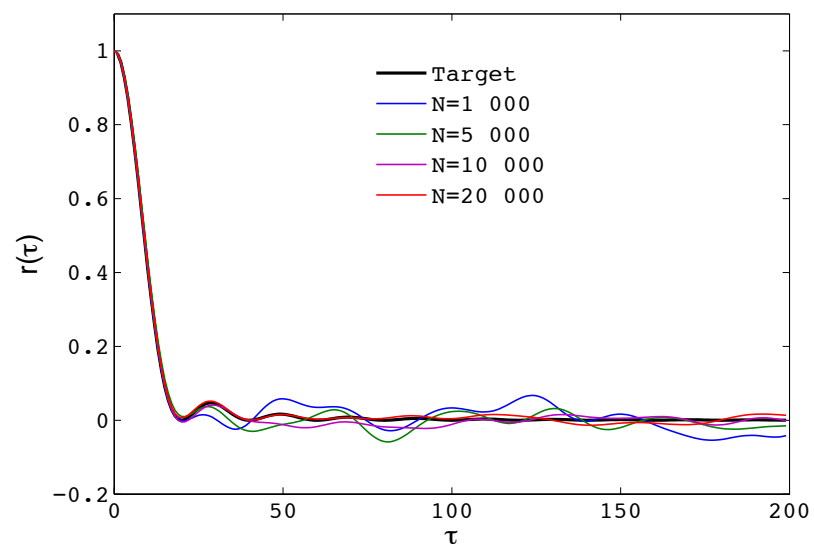

Figure 6: Convergence of the correlation function estimate with respect to the number of Monte Carlo simulations for $p^{\text {sim }}=512$.

It is seen that the order of expansion has to be choosen such that $p^{\text {sim }} \geqslant 256$. The value $p^{\text {sim }}=512$ was then used in the simulations. An example of the estimated mesoscopic correlation function is plotted on Fig. (8). The graph of mapping $l_{\mathbf{U}}^{x} \rightarrow l_{\mathbf{C}}^{x}$, estimated from 20000 Monte Carlo simulations, is plotted on Fig. (9). For a given direction, the relation between the correlation lengths of the stochastic germs and the mesoscopic random field

\footnotetext{
${ }^{2}$ It can be shown that in the present case, $p^{\text {sim }}$ is a power of 2 .
} 


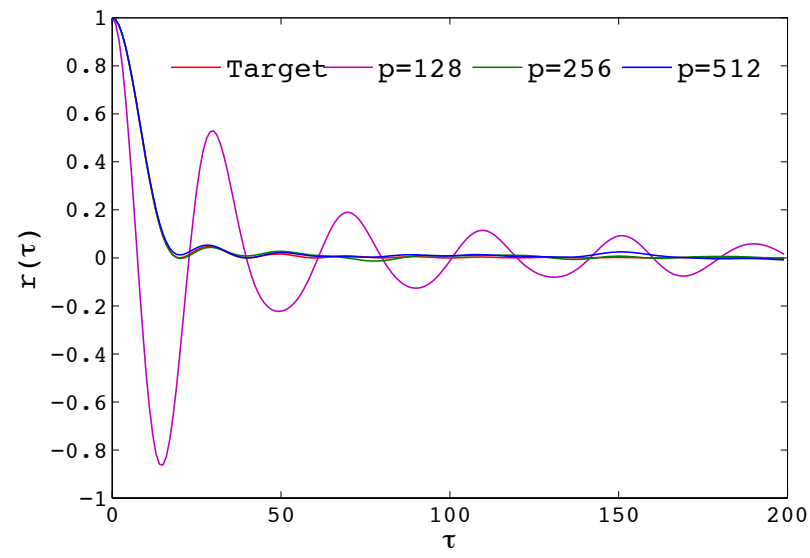

Figure 7: Convergence of the correlation function estimate with respect to the order $p^{\text {sim }}$.

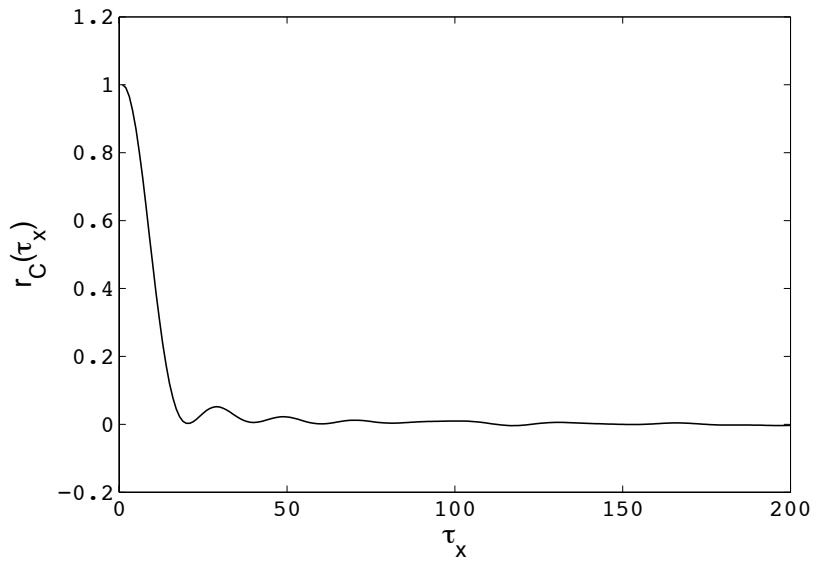

Figure 8: Graph of function $\tau_{x} \rightarrow r_{\mathbf{C}}\left(\tau_{x}\right)$ estimated for $l_{\mathbf{U}}^{x}=9.76 \mathrm{~mm}$.

can then be approximated as linear:

$$
l_{\mathbf{C}}^{k} \approx 1.1 l_{\mathbf{U}}^{k}
$$

where $k$ stands either for $x$ or $y$. Finally, Eq. (26) together with Eqs. (24) and (25) allow the spatial correlation lengths of the stochastic germs to be identified:

$$
l_{\mathbf{U}}^{x}=10.8 \mathrm{~mm}
$$




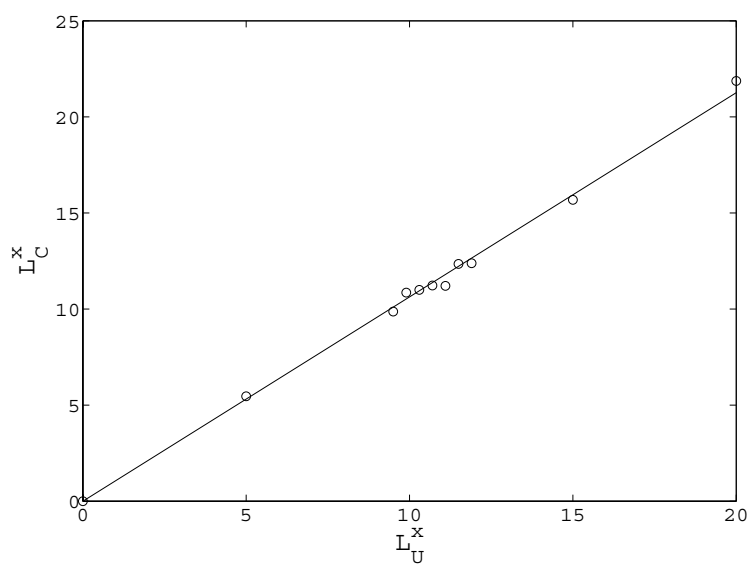

Figure 9: Graph of the mapping $l_{\mathbf{U}}^{x} \rightarrow l_{\mathbf{C}}^{x}$.

$$
l_{\mathbf{U}}^{y}=7.5 \mathrm{~mm}
$$

\subsubsection{Identification of dispersion parameter}

Since experimental trajectories of the random fields are known, the dispersion parameter could be identified from the following estimate:

$$
\widehat{\delta}_{\mathbf{C}}=\frac{1}{\|\underline{\widehat{\mathbf{C}}}\|_{\mathrm{F}}^{2}}\left\{\frac{1}{N^{e x p} p^{e x p}} \sum_{i=1}^{N^{e x p}} \sum_{j=1}^{p^{e x p}}\left\|\mathbf{C}^{\exp }\left(\mathbf{x}_{i}, \theta_{j}\right)-\underline{\widehat{\mathbf{C}}}\right\|_{\mathrm{F}}^{2}\right\}^{1 / 2}
$$

This yields:

$$
\widehat{\delta}_{\mathbf{C}}=6.7 \cdot 10^{-3} \text { and } \widehat{\delta}=9.4 \cdot 10^{-3}
$$

It is seen that the direct use of a statistical estimate results in very small values of the dispersion parameters $\delta_{\mathbf{C}}$ and $\delta$. This is because the parameter not only controls the level of the statistical fluctuations, but also, in a sense, the degree of anisotropy of the microstructure. Then, identifying the parameter by considering locally isotropic realizations provides an underestimation of the dispersion parameter. In order to tackle this difficulty, we propose the following methodology. Let $\mathbb{C} \rightarrow \Delta_{\mathbf{C}}(\mathbb{C})$ be the function defined from $\mathbb{M}_{n}^{+}(\mathbb{R})$ into $\mathbb{R}^{+}$by:

$$
\Delta_{\mathbf{C}}(\mathbf{C})=\frac{1}{|\Omega|} \int_{\Omega}\left\{\frac{E\left\{\|\mathbf{C}(\mathbf{x})-\underline{\mathbf{C}}(\mathbf{x})\|_{\mathrm{F}}^{2}\right\}}{\|\underline{\mathbf{C}}(\mathbf{x})\|_{\mathrm{F}}^{2}}\right\}^{1 / 2} d \mathbf{x}
$$


This function allows to characterize the level of statistical fluctuations of random field $\mathbf{x} \rightarrow \mathbf{C}(\mathbf{x})$ over the domain $\Omega$. It should be noted that when applied to the elasticity tensor random field defined in section (2.1), in the homogeneous case, Eq. (31) yields $\Delta_{\mathbf{C}}(\mathbf{C})=\delta_{\mathbf{C}}$. The idea is to choose the dispersion parameter such that the level of fluctuations (estimated from Eq. (31)) of the tensor $\mathbf{C}^{*}$ whose components are extracted from the anisotropic tensor $\mathbf{C}$ and are such that $\mathbf{C}^{*}(i, j)=\mathbf{C}(i, j)$ iff $1 \leq i, j \leq 3$ or $i=j \in$ $\{4,5,6\}\left(\mathbf{C}^{*}(i, j)=0\right.$ otherwise $)$, is the same as the level of fluctuations of the experimental realizations. When the mean matrix and the spatial correlation lengths of the stochastic germs are fixed (in the sequel, they are defined by the experimental values (23), (27) and (28)), the mapping $\delta \rightarrow$ $\Delta_{\mathbf{C}}\left(\mathbf{C}^{*}\right)$ can be numerically computed. The plot of this function is shown on Fig. (10), where the relation considering the initial anisotropic random field is also depicted. It is seen that while the two mappings obviously digress

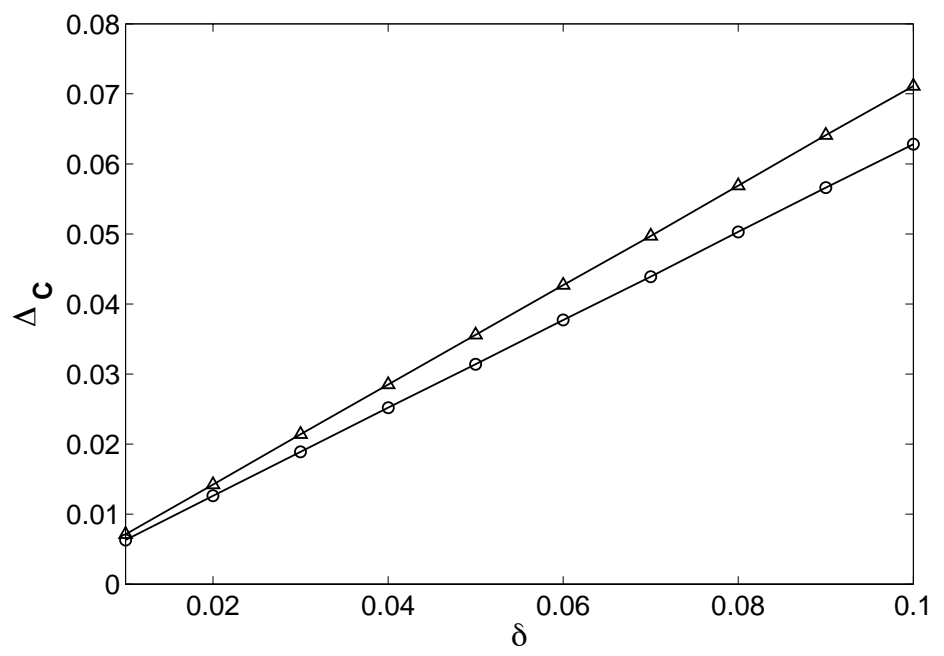

Figure 10: Graphs of the mapping $\delta \rightarrow \Delta_{\mathbf{C}}$ : (i) extracted random field $\mathbf{x} \rightarrow \mathbf{C}^{*}$ (circle) (ii) anisotropic random field $\mathbf{x} \rightarrow \mathbf{C}$ (triangle-up).

as $\delta$ increases, the difference between the two estimates remains quite small because of the isotropy of the mean matrix. The function $\delta \rightarrow \Delta_{\mathbf{C}}\left(\mathbf{C}^{*}\right)$ is then shown to be linear and such that: $\Delta_{\mathbf{C}}=0.62782 \cdot \delta$. Next, applying Eq. (31) to the experimental realizations yields: $\Delta_{\mathbf{C}}\left(\mathbf{C}^{\exp }\right)=0.0595$. Finally, it can be deduced that:

$$
\widehat{\delta}_{\mathbf{C}}^{*}=0.0674 \text { and } \widehat{\delta}^{*}=0.0948
$$


The probabilistic model associated to the considered microstructure is then completely defined by Eqs. (23), (27), (28) and (32).

\subsection{Probabilistic properties of the identified volume fraction random field} and computational identification

\subsubsection{Properties of the experimental trajectories}

As a first result, kernel estimates of four experimental marginal density functions are plotted on Fig. (11). All the experimental marginal densities

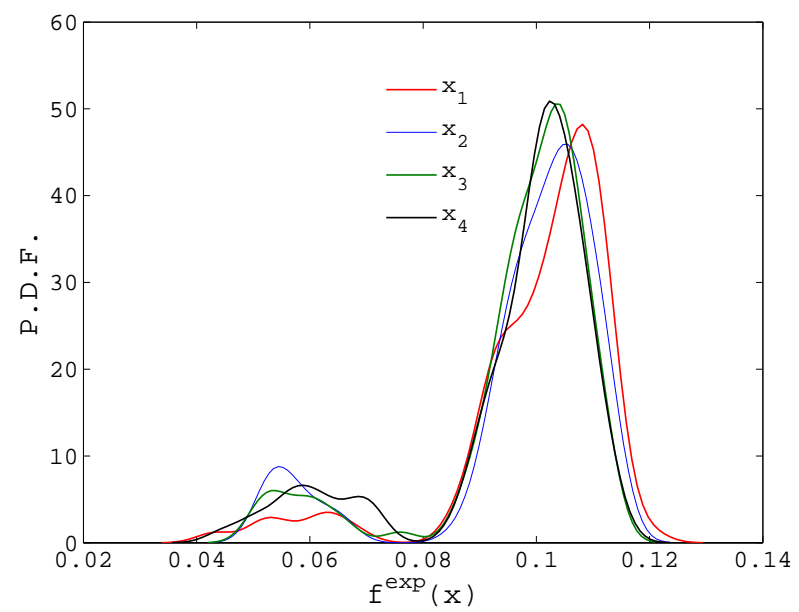

Figure 11: Kernel estimates of the experimental marginal density functions in $\mathbf{x}_{1}, \mathbf{x}_{2}, \mathbf{x}_{3}$ and $\mathbf{x}_{4}$.

appear to be bimodal. A possible origin of this bimodality (and in particular, of the first mode) is that the thickness of the plates was assumed to be homogeneous and constant in the experimental analysis. However, small fluctuations were observed in practice and the thickness value retained in the computation can be considered as an upper bound (corresponding to the thickness of the injection mould), leading in some locations to the underestimation of the longitudinal velocity and thus, of the volume fraction.

Since no boundary effect on the experimental trajectories was observed, we assume that random field $\mathbf{x} \rightarrow f(\mathbf{x})$ can be considered as the restriction to $\Omega$ of a homogeneous random field. As a result, the mean function of $f$ does not depend on $\mathbf{x}$ anymore and correlation between two points only 
depends on the distance between them. The consequence of this approximation on the mean function can be illustrated by considering the function $\mathbf{x} \rightarrow \epsilon^{\operatorname{Smod}}(\mathbf{x})=1-\underline{f}(\mathbf{x}) / \underline{f}$ (where $\underline{\hat{f}}$ denotes the overall mean value computed over all locations) whose graph is reported on Fig. (12). It is seen

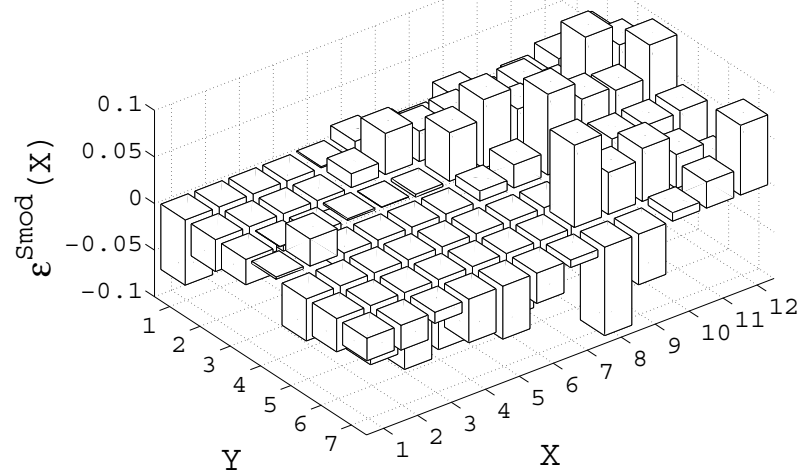

Figure 12: Graph of $\mathbf{x} \rightarrow \epsilon^{\operatorname{Smod}}(x)$.

that the mean function can be reasonably considered as constant over the composite plate, with an overall mean value $\widehat{f}=0.0936$ very close to the target value $f^{\text {tgt }}(=10 \%)$. Moreover, based on this assumption, the mean ergodicity assumption can be readily tested. We recall that this assumption consists in substituting the spatial mean estimate, computed for a given realization, for the statistical mean estimate. The convergence of the spatial mean estimate for the first ten experimental realizations is depicted on Fig. (13). It is seen that spatial estimates basically reach convergence for a rather small number of sampling points (around 60). The convergence for the 110 experimental realizations, as well as the probability density function of the converged estimates, are depicted on Figs. (25) and (26) in Appendix B. Moreover, the coefficient of variation of the spatial mean estimate (computed from the 110 converged values) is about $5.7 \%$. Consequently, the mean ergodicity assumption may seem acceptable in the present case, depending on whether or not the value of the coefficient of variation is considered as negligible. 


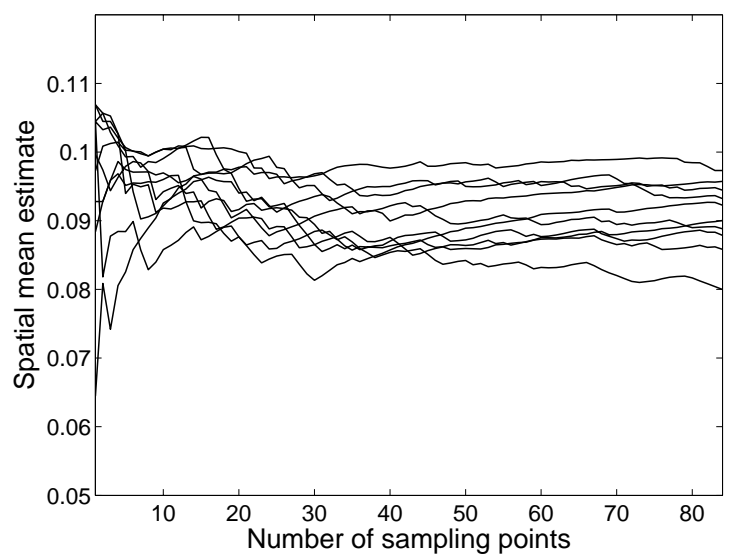

Figure 13: Convergence of the spatial mean estimate for the first ten experimental realizations of the random field.

\subsubsection{Statistical reduction and Projection}

Making use of a collocation method, Eq. (8) can be converted into a classical eigenvalue problem which can be numerically solved. The graph of the first eigenfunction is plotted on Fig. (14). An optimal order of expansion

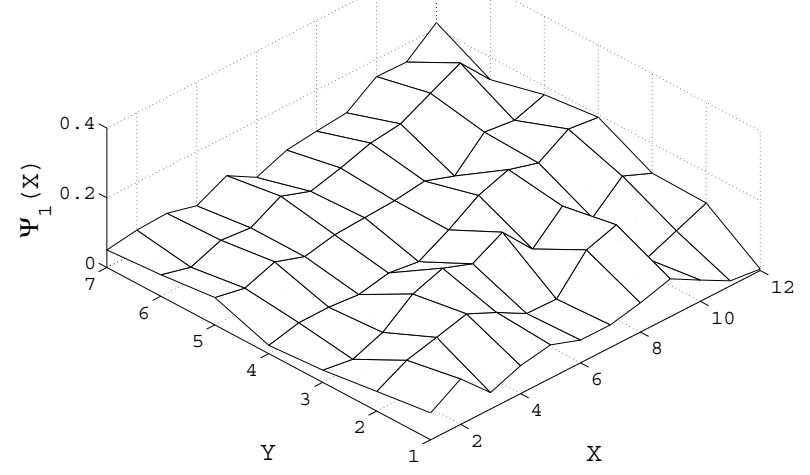

Figure 14: Graph of the first eigenfunction $\mathbf{x} \rightarrow \psi_{1}(\mathbf{x})$.

in the KL-representation (see Eq. (7)) can be determined by characterizing the convergence of the function $p \rightarrow \epsilon^{\operatorname{Conv}-\mathrm{KL}}(p)=1-\sum_{i=1}^{p} \lambda_{i} / \sum_{i=1}^{N^{\exp }} \lambda_{i}$, 
where $N^{\exp }$ is the number of experimental sampling points $\left(N^{\exp }=84\right)$. As shown in $[6]$, one has $\epsilon^{\mathrm{Conv}-\mathrm{KL}}(41) \approx 0.1$ in the present case and then, $M=41$ (see Appendix C). It should be noted that because of the experimental analysis, this value is much higher than typical values used in the litterature (a $5^{\text {th }}$-order approximation was used in [2] in the case of a fully simulated database, for instance).

The parallel computations dedicated to the random search procedure (involved in the identification of the chaos coefficients, see section (2.2)) have been performed on a 8 -node cluster $\left(10^{8}\right.$ matrices have been tested). Results are illustrated on Figs. (15) and (16), where the convergence of the marginal densities for random variables $\eta_{1}$ and $\eta_{6}$ and with respect to the order of expansion has been reported (for $m=5$ ). As expected, the sim-

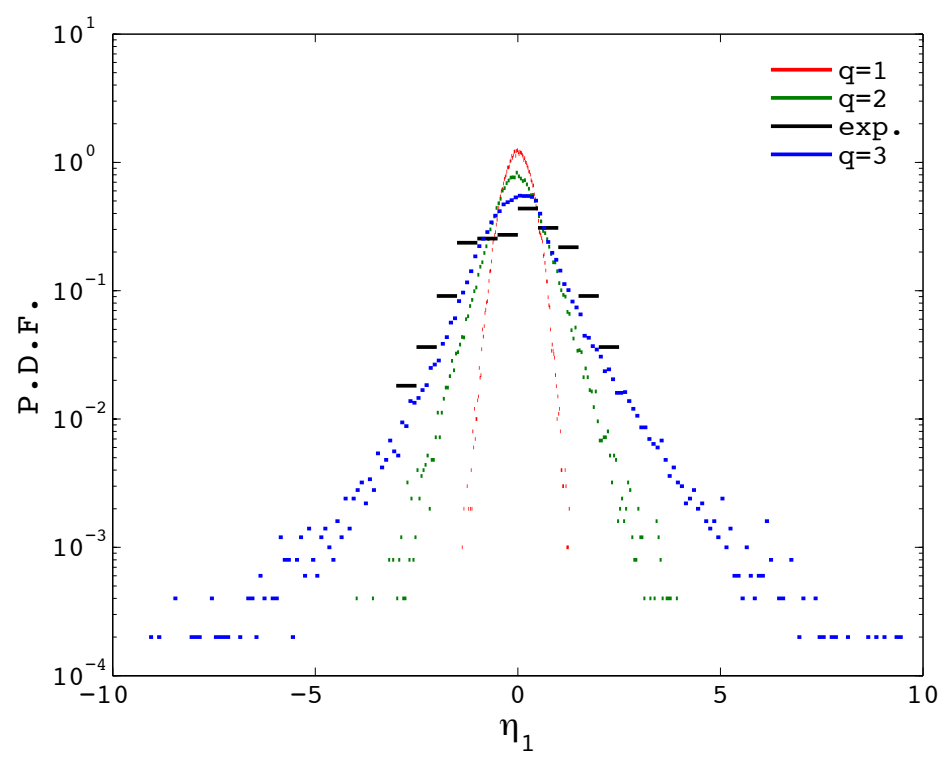

Figure 15: Plot of the experimental and simulated marginal density function of $\eta_{1}$ for different order of Chaos expansion.

ulated marginal densities converge towards the experimental ones as the order of the representation $q$ increases. Moreover, it is seen that a third order approximation yields accurate results, so that the final representation 


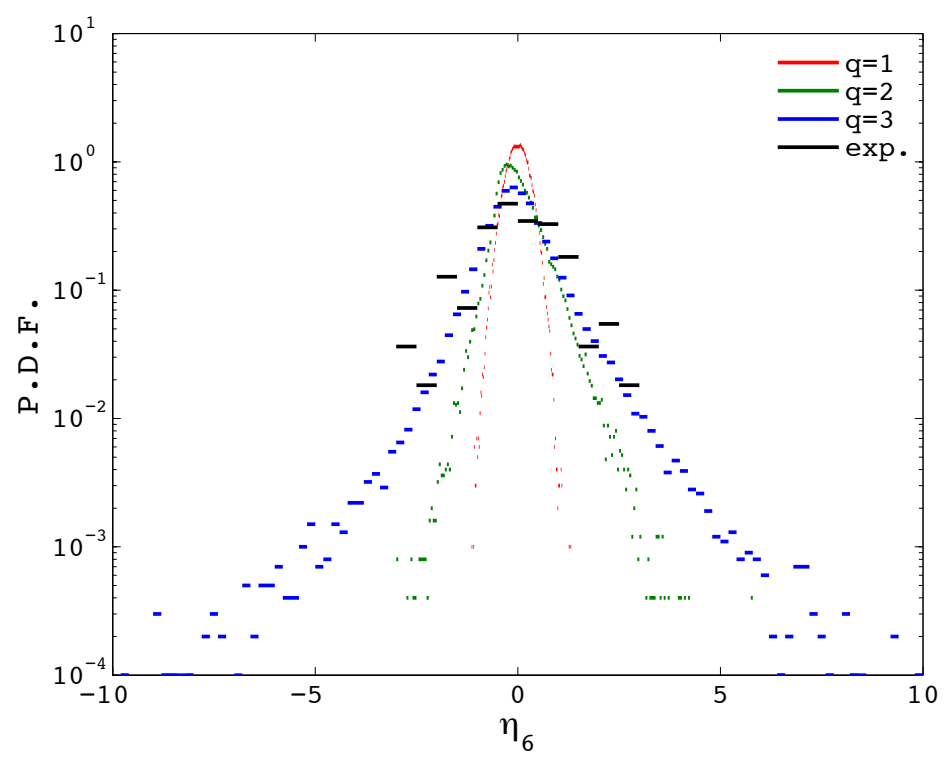

Figure 16: Plot of the experimental and simulated marginal density function of $\eta_{6}$ for different order of Chaos expansion.

of random field $\mathbf{x} \rightarrow f(\mathbf{x})$ can be written as:

$$
f(\mathbf{x})=\underline{f}+\sum_{k=1}^{41} \sqrt{\lambda_{k}}\left\{\sum_{\gamma \in \mathbb{N}^{m},|\gamma|=1}^{3} z_{k}^{\gamma} \tilde{H}_{\gamma}(\xi)\right\} \psi_{k}(\mathbf{x})
$$

In practice, numerical Monte Carlo simulations of the Gaussian germ $\xi$, together with Eq. (33), allow trajectories of the random field to be simulated. A comparison between experimental and simulated (with 50000 simulated realizations of $\xi$ ) marginal densities is provided on Figs. (17) and (18), for points $\mathbf{x}_{1}$ and $\mathbf{x}_{2}$ respectively. Since the stationary approximation may introduce a shift in the mean value, note that the graphs have been centered in order to make the comparison of the statistical fluctuations easier.

It is seen that while there is a good match between the experimental data and the simulated realizations, the bimodality is not predicted by the probabilistic model (and in particular, by the chaos expansion). This result may be explained by noticing that the experimental marginal distributions of random variables $\eta_{i}$ are unimodal (see Figs. (15) and (16)). 


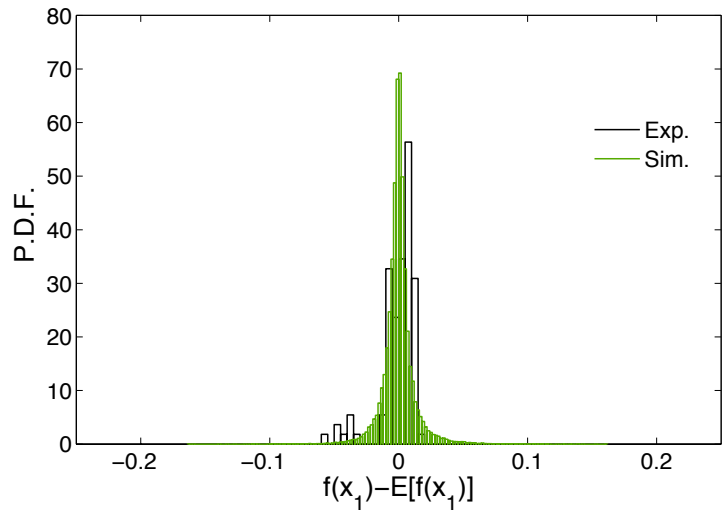

Figure 17: Comparison between the experimental and the model-based simulated marginal density function at $\mathbf{x}_{1}$.

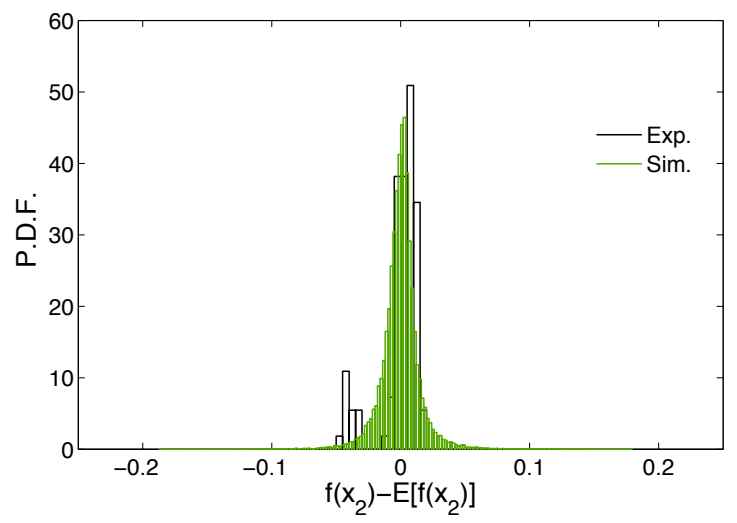

Figure 18: Comparison between the experimental and the model-based simulated marginal density function at $\mathbf{x}_{2}$.

\section{Probabilistic parametric analysis of the RVE size}

We now illustrate the use of the stochastic mesoscopic modeling in the definition of the RVE size. For this purpose, we will consider the probabilistic model introduced in section (2.1) and simulated with the parameters identified in the previous section. 
Let $e_{i}(\theta)$ be the random norm defined by:

$$
e_{i}(\theta)=\left\|\widetilde{\mathbf{C}}_{i}(\theta)\right\|=\sup _{\|\mathbf{x}\| \leq 1}\left\|\widetilde{\mathbf{C}}_{i}(\theta) \mathbf{x}\right\|, \mathbf{x} \in \mathbb{R}^{6}
$$

where $\widetilde{\mathbf{C}}_{i}(\theta)$ is the apparent (random) matrix computed for realization $\theta$ and considering a volume $V_{i}$ containing $i^{2}$ mesovolumes (see Fig. (19), where the definition of the growing volumes is depicted). Following [23], a probabilistic

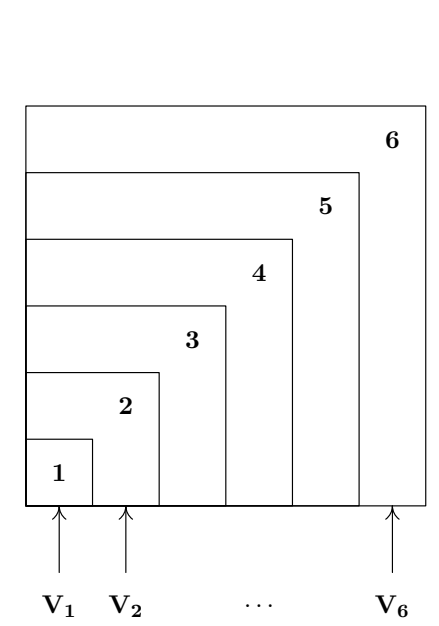

Figure 19: Schematic of the size increase for the parametric analysis of the RVE size.

convergence analysis can be performed by considering the convergence of the mapping $i \rightarrow C V\left[e_{i}\right]$, where $C V[z]$ denotes the coefficient of variation of random variable $z$. Since we are more interested in characterizing the global probabilistic convergence rather than on an accurate estimation of the apparent elasticity tensor, the realizations of the overall tensors are computed by using a simple Voigt estimate, that is to say:

$$
\widetilde{\mathbf{C}}_{i}(\theta)=\frac{1}{i^{2}} \sum_{j=1}^{i^{2}} \mathbf{C}\left(\mathbf{x}_{j}, \theta\right)
$$

The convergence of the statistical estimate of the coefficient of variation (of the random norm) with respect to the number of Monte Carlo simulations is depicted on Fig. (20). It is seen that the convergence is reached for 500 simulations. Next, the convergence of the coefficient of variation with respect to the size of the volume under investigation is reported on Fig. 


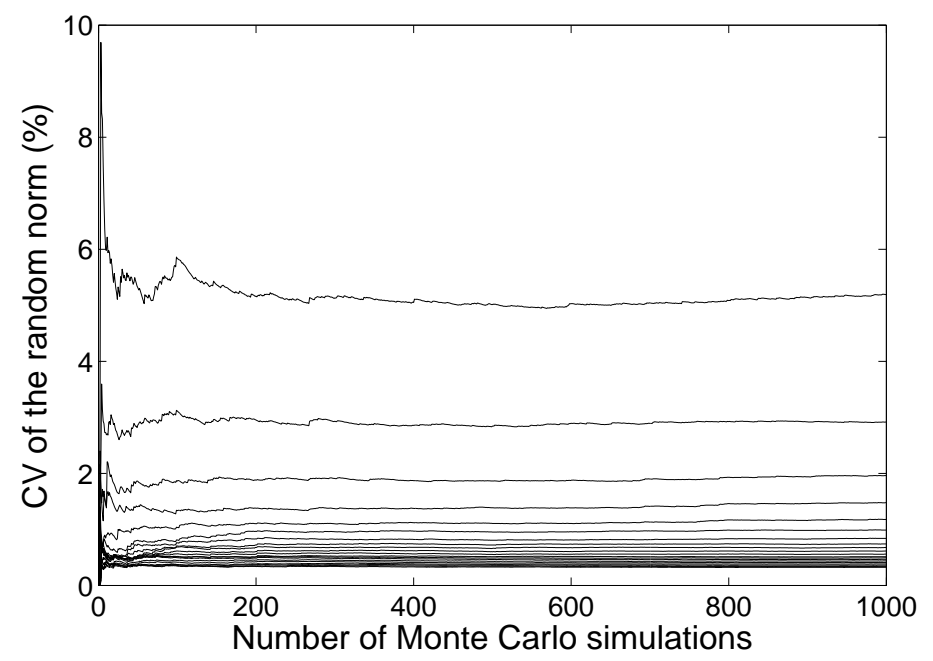

Figure 20: Convergence of the coefficient of variation of the random norm with respect to the number of Monte Carlo simulations, for all configurations of mesovolumes.

(21). It is seen that the coefficient of variation is less than $1 \%$ (resp. $0.5 \%$ ) when the volume contains $6^{2}=36$ (resp. $13^{2}=169$ ) mesovolumes. In order to improve this very first parametric analysis, let us consider the normalized random variable:

$$
Z=\frac{e_{i}}{E\left[e_{i}\right]}
$$

Let $\beta \rightarrow \mathrm{P}(\beta)$ be the function defined by:

$$
\mathrm{P}(\beta)=P\{1-\beta<Z \leq 1+\beta\}=E\left\{\mathbf{1}_{Z \leq 1+\beta}\right\}-E\left\{\mathbf{1}_{Z \leq 1-\beta}\right\}
$$

where $\mathbf{1}_{Z \leq z^{*}}=1$ if $Z \leq z^{*},=0$ otherwise. The graphs of function $\beta \rightarrow \mathrm{P}(\beta)$ for different sizes of the considered volume are shown on Fig. (22). For instance, in the case of a volume containing 25 mesovolumes (see the dashed line on Fig. (22)), it is seen that the level of statistical fluctuations is respectively less than $2 \%, 2.4 \%$ and $3.1 \%$ with probability $0.9,0.95$ and 0.99. From these results, it is then possible to extract the convergence of the level of statistical fluctuations for a given probability level: such a convergence is illustrated on Fig. (23), for probability 0.9, 0.95 and 0.99 .

From Fig. (23), it can be deduced that if one defines the RVE size with respect to a level of fluctuations less than $1 \%$, then the characteristic size (in one direction) of the RVE is about 10 (resp. 12 and 16) times the size 


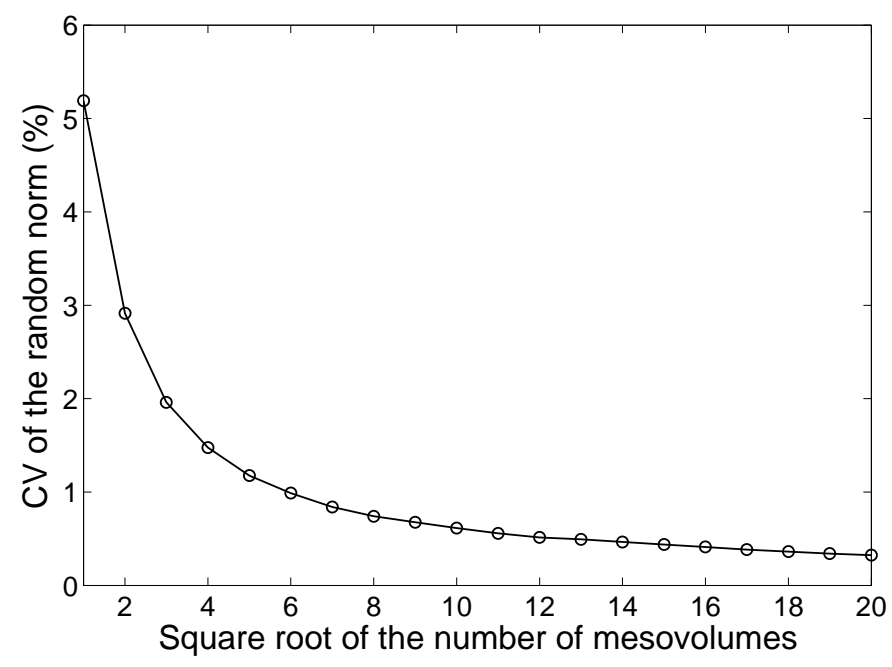

Figure 21: Convergence of the coefficient of variation of the random norm with respect to the size of the volume under investigation.

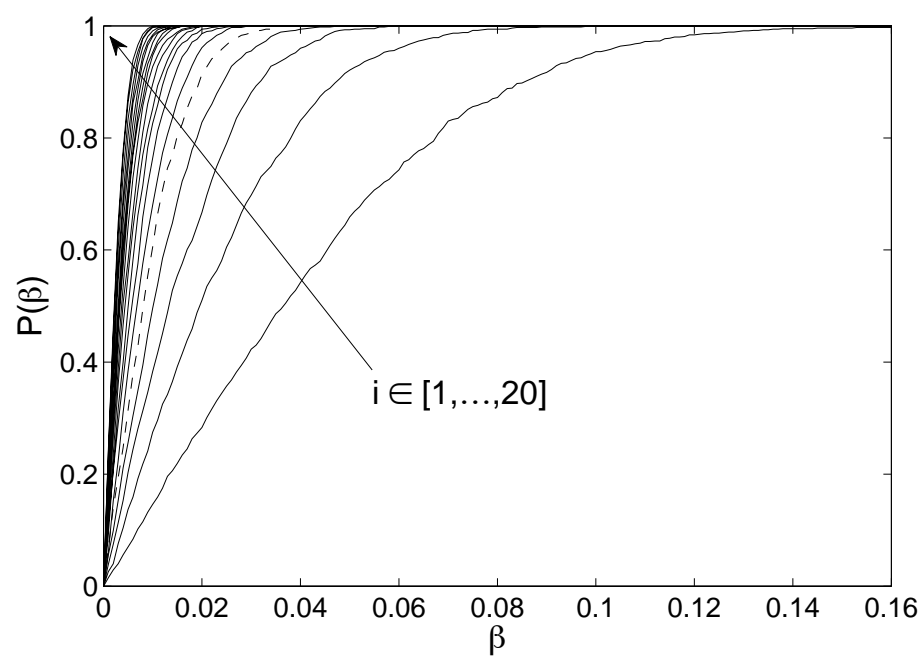

Figure 22: Graphs of $\beta \rightarrow \mathrm{P}(\beta)$ for volumes containing $i^{2}$ mesovolumes, $i \in[1, \ldots, 20]$ (the dashed line corresponds to the volume $V_{5}$ ). 


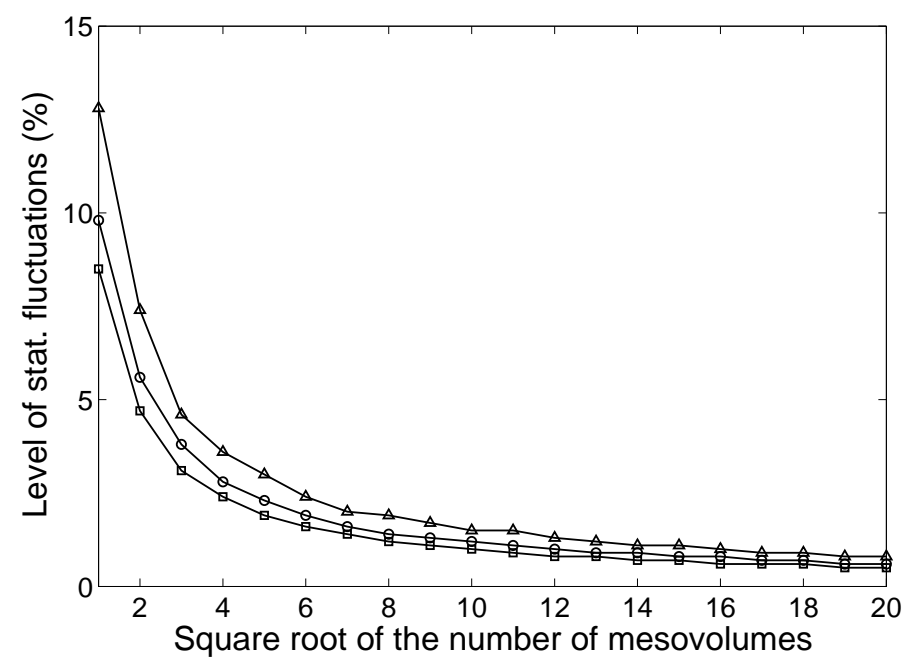

Figure 23: Convergence of the level of statistical fluctuations with respect to the size of the considered volume, for probability 0.9 (square marker), 0.95 (circle) and 0.99 (triangle-up).

of a mesovolume with probability 0.9 (resp. 0.95 and 0.99 ). These ratios can also be expressed in terms of the spatial correlation lengths and remain approximately the same, since the correlation lenghts are roughly equal to the size of a mesovolume. These orders of magnitude for the definition of the RVE size are in agreement with the ones provided in [23] and [27].

\section{Conclusion}

This paper is devoted to the computational and experimental identification of two probabilistic models that can be used for modelling the elasticity tensor random field at the mesoscale of fiber reinforced composites. These two models were first recalled. Then, a innovative methodology allowing the experimental trajectories of the volume fraction and elasticity tensor random fields to be identified was introduced and is based on the combination of an ultrasonic testing with the formulation and numerical resolution of an inverse (micro-)mechanical problem.

The parameters of the probabilistic model for the elasticity tensor random field were identified by using either a statistical estimate (for the mean matrix) or a stochastic optimization algorithm (for the mesoscopic spatial correlation lenghts). Based on these estimations, the correlation lengths of 
the stochastic germs were identified in turn. A methodology to estimate physically consistent dispersion parameters was also introduced. All these identified parameters allow trajectories of the stiffness tensor random field to be easily simulated and may be used as a reference for uncertainty propagation (when using a Stochastic Finite Elements Method for instance).

Considering the mesoscopic volume fraction, the random field was considered as the restriction of a homogeneous random field, allowing the mean ergodicity assumption to be tested. In particular, the scatter resulting from all the spatial mean estimates was characterized and can be used to discuss the relevance of such an assumption. Next, the parameters involved in the model (that are, the coefficients of the Chaos expansion) were identified making use of the Maximum Likelihood Principle. Simulated marginal densities (with a third order Chaos expansion) are then shown to be in agreement with the experimental ones, demonstrating the relevance of both the probabilistic model and strategy of identification.

Finally, the use of such models in order to investigate the size of the RVE is illustrated through a probabilistic parametric analysis. It is shown that the characteristic length of the macroscale may be defined with respect to the mesoscopic correlation lengths, depending on the choice of an admissible level of statistical fluctuations. The order of magnitude for the definition of the RVE size is in agreement with values provided in the literature.

\section{Acknowledgements}

Professor C. Binetruy is gratefully acknowledged for having partly provided the computational and experimental ressources that were used in this study. 


\section{A. Mapping between the velocity and the volume fraction}

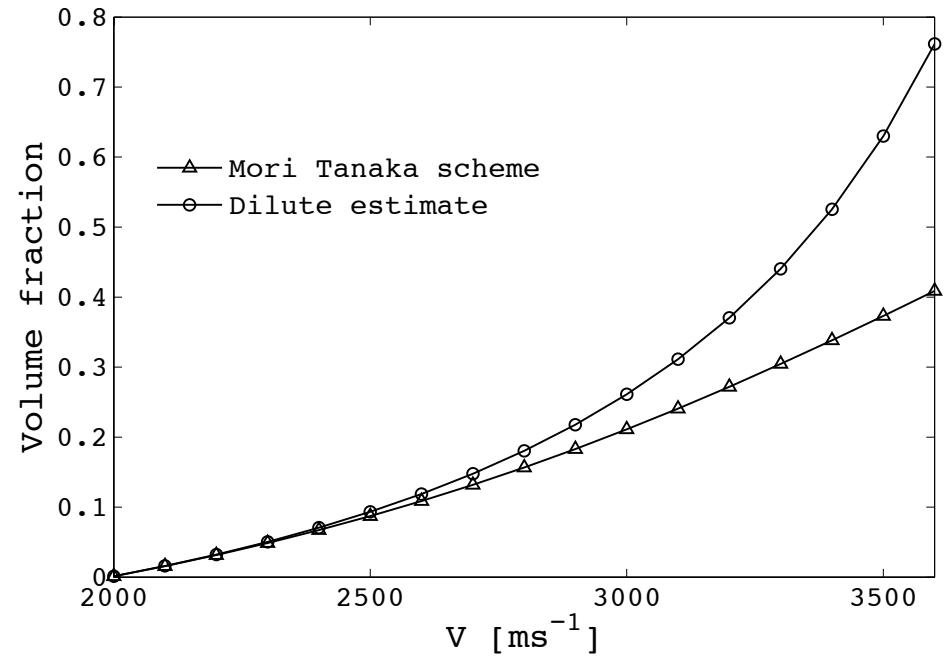

Figure 24: Plot of the mapping $V_{l} \rightarrow f=\Lambda^{-1}\left(V_{l}\right)$ computed by using the Mori-Tanaka and dilute estimates (see [6]). 


\section{B. Convergence analysis of the spatial mean estimate}

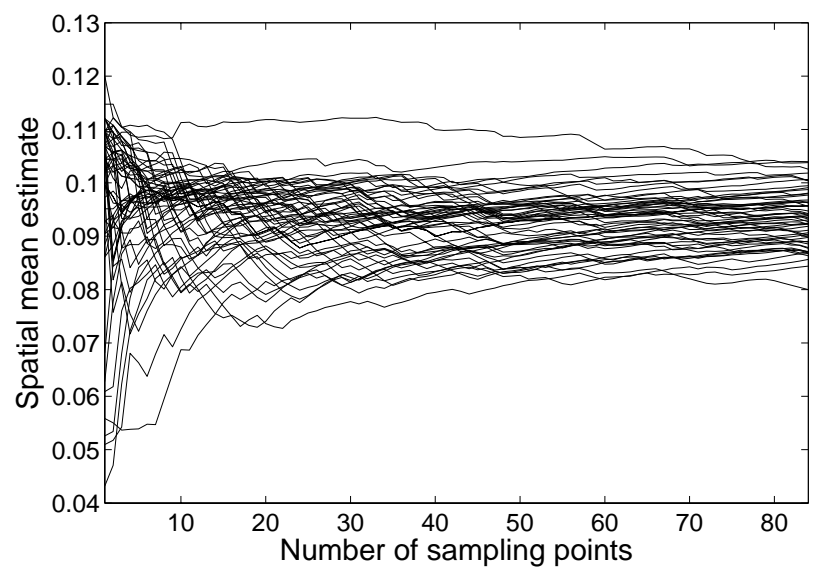

Figure 25: Convergence of the spatial mean estimate for the 110 experimental realizations of the elasticity tensor random field.

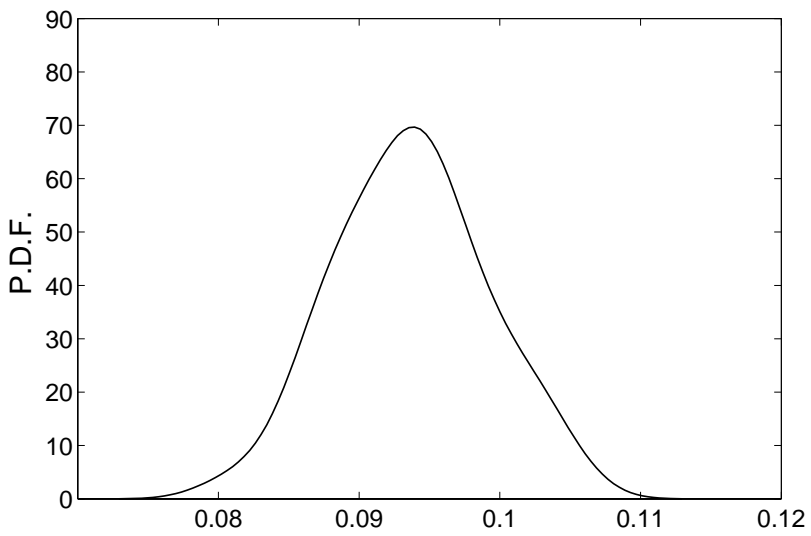

Figure 26: Kernel estimate of the probability density function of the converged spatial mean estimates. 
C. Error arising from the KL expansion truncature

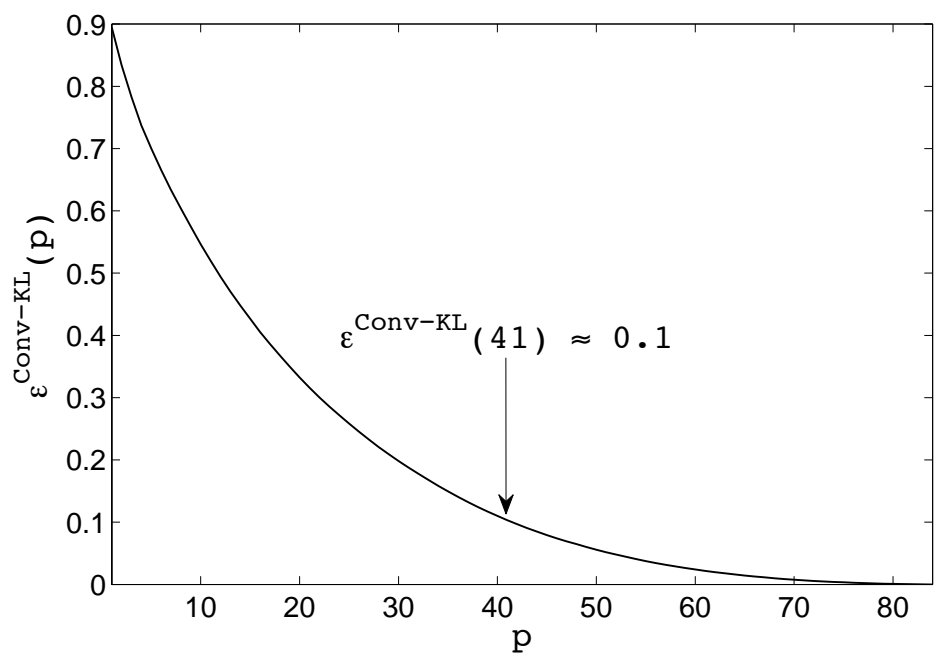

Figure 27: Graph of function $p \rightarrow \epsilon^{\text {Conv-KL }}(p)$. 


\section{References}

[1] S. C. Baxter, M. I. Hossain, and L. L. Graham. Micromechanics based random material property fields for particulate reinforced composites. International Journal of Solids and Structures, 38:9209-9220, 2001.

[2] C. Desceliers, R. Ghanem, and C. Soize. Maximum likelihood estimation of stochastic chaos representations from experimental data. Int. J. Numer. Meth. Engng, 66:978-1001, 2006.

[3] R. Ghanem and P. Spanos. Stochastic finite elements: a Spectral Approach. Springer, New-York, 1991.

[4] L. L. Graham, S.R. Arwadea, D.J. Corrb, M.A. Gutierrez, D. Breyssed, M. Grigoriu, and N. Zabaras. Probability and materials. from nano- to macro-scale: A summary. Prob. Eng. Mechanics, 21(3):193-199, 2006.

[5] L. L. Graham and S. C. Baxter. Simulation of local material properties based on moving-window gmc. Prob. Eng. Mechanics, 16:295-305, 2001.

[6] J. Guilleminot, C. Soize, D. Kondo, and C. Binetruy. Theoretical framework and experimental procedure for modelling mesoscopic volume fraction stochastic fluctuations in fiber reinforced composites. International Journal of Solids and Structures, 45:5567-5583, 2008.

[7] C. Huet. Application of variational concepts to size effects in elastic heterogeneous bodies. Journal of the Mechanics and Physics of Materials, 38(6):813-841, 1990.

[8] E. T. Jaynes. Information theory and statistical mechanics. Physical Review, 106(4):620-630, 1957.

[9] E. T. Jaynes. Information theory and statistical mechanics. Physical Review, 108(2):171-190, 1957.

[10] D. Jeulin. Morphological characterization and models of random structures. In Homogenization in Mechanics of Materials. ISTE Ltd. and John Wiley \& Sons, 2009.

[11] R. M. Lewis and V. Torczon. Pattern search algorithms for bound constrained minimization. SIAM J. Optim., 9(4):1082-1099, 1999.

[12] R. M. Lewis and V. Torczon. A globally convergent augmented lagrangian pattern search algorithm for optimization with general constraints and simple bounds. SIAM J. Optim., 12(4):1075-1089, 2002. 
[13] M. Loève. Probability Theory, 4th Ed. Springer Verlag, New-York, 1977.

[14] M. Ostoja-Starzewski. Microstructural Randomness and Scaling in Mechanics of Materials. Chapman \& Hall-CRC-Taylor \& Francis, 2008.

[15] F. Poirion and C. Soize. Numerical methods and mathematical aspects for simulation of homogeneous and nonhomogeneous gaussian vector fields. In W. Kre and W. Wedig, editors, Probabilistic Methods in Applied Physics, Lecture Notes in Physics, pages 17-53. Springer-Verlag, Berlin, Heidelberg, 1995.

[16] K. Sab. On the homogenization and the simulation of random materials. European Journal of Mechanics A/Solids, 11(5):585-607, 1992.

[17] R. J. Serfling. Approximation Theorems of Mathematical Statistics. John Wiley \& Sons, 1980.

[18] J. Serra. Image analysis and Mathematical Morphology. Academic Press, London, 1982.

[19] C. E. Shannon. A mathematical theory of communication. Bell System Tech. J., 27:379-423/623-659, 1948.

[20] M. Shinozuka. Simulations of multivariate and multidimensional random processes. J. Acoust. Soc. Amer., 39(1):357-367, 1971.

[21] B. W. Silverman. Density Estimation for Statistics and Data Analysis. Chapman \& Hall CRC, 1986.

[22] C. Soize. Non-gaussian positive-definite matrix-valued random fields for elliptic stochastic partial differential operators. Computer Methods in Applied Mechanics and Engineering, 195:26-64, 2006.

[23] C. Soize. Tensor-valued random fields for meso-scale stochastic model of anisotropic elastic microstructure and probabilistic analysis of representative volume element size. Prob. Eng. Mechanics, 23:307-323, 2008.

[24] C. Soize and R. Ghanem. Physical systems with random uncertainties: Chaos representations with arbitrary probability measure. SIAM Journal on Scientific Computing, 26(2):395-410, 2004.

[25] S. Torquato. Random Heterogeneous Materials: Microstructure and Macroscopic Properties. Springer, New-York, 2002. 
[26] N. Wiener. The homogeneous chaos. American Journal of Mathematics, 60:897-936, 1938.

[27] X. F. Xu and X. Chen. Stochastic homogenization of random elastic multi-phase composites and size quantification of representative volume element. Mechanics of Materials, 41:174-186, 2009. 